\title{
On the Asymptotic Performance of Multiple Antenna Channels with Fast Channel Feedback
}

\author{
Ahmad Khoshnevis and Ashutosh Sabharwal
}

\begin{abstract}
In this paper, we analyze the asymptotic performance of multiple antenna channels where the transmitter has either perfect or finite bit channel state information. Using the diversitymultiplexing tradeoff to characterize the system performance, we demonstrate that channel feedback can fundamentally change the system behavior. Even one-bit of information can increase the diversity order of the system compared to the system with no transmitter information. In addition, as the amount of channel information at the transmitter increases, the diversity order for each multiplexing gain increases and goes to infinity for perfect transmitter information. The major reason for diversity order gain is a "location-dependent" temporal power control, which adapts the power control strategy based on the average channel conditions of the channel.
\end{abstract}

\section{Introduction}

The importance of channel state information at transmitter has been extensively studied, see for example [1-7]. In fading channels, channel state information at the transmitter leads to significant gains in outage probability over systems with no information at the transmitter. There are many widely used methods to exploit the transmitter channel information like power control and beamforming. However, the comparative performance of these different methods is still unclear. In this paper, we characterize the diversity order in systems with perfect or imperfect channel state information at the transmitter, with the aim of identifying techniques which yield maximum benefit in outage performance.

We study systems in which the transmitter has causal channel information and adapts its actions based on the current channel conditions. Our main contributions are four-fold. First, we study the performance with perfect channel information at the transmitter and receiver, and show that the diversity order of optimal power control is infinite at all multiplexing gains, for many antenna configurations. To enable fair comparisons with systems which have no or imperfect channel information at the transmitter, the outage definition is generalized to include instances in which no information is transmitted by the sender. Thus, the outage event contains all the cases in which either the transmitter sends no information or the information is sent but corrupted by the channel.

Second, we refine our analysis further by decoupling the optimal power control into temporal power control and spatial power control. In temporal power control, all eigenvectors of the channel receive the same equal power, with total power depending on the current channel conditions. And in spatial power control, a short term power constraint is employed leading to same total power for each channel condition but adaptive power allocation along different eigenvectors of the channel. We show that most of the gain of optimal power control stems from temporal power control. In fact, 
spatial power control has the same diversity-multiplexing tradeoff as the system with no channel information at the transmitter. To achieve the gain from temporal power control, an adaptive power control is critical which adapts based on average condition of forward channel.

Third, we derive lower bounds on diversity order for systems where only finite number of feedback bits about the channel are made available to the transmitter. The analysis is limited to only temporal power control since only temporal power control provides increase in diversity (as shown by the above mentioned perfect channel information analysis). To aid closed form analysis, we develop a simple finite bit quantizer which can be computed in a recursive manner. We show that finite bit channel information leads to a substantial (though finite) increase in diversity order for each multiplexing gain, when compared to system with no channel information [8]. And as the number of feedback bits increase, the diversity order increases unboundedly for all but the maximum multiplexing gain, and approaches the perfect information case. The proof of the finite rate feedback relies on approximations of order statistics of eigenvalues of Gaussian matrices, which are of independent interest.

Fourth and last, we also consider joint rate and power control with finite number of feedback bits, and show that a non-zero diversity gain is possible for highest multiplexing gain even with imperfect transmitter information. This contrasts the temporal power control methods with finite rate feedback which have zero diversity order for full multiplexing gain.

Several recent works $[9,10]$ have derived the diversity-multiplexing tradeoff with channel information at the transmitter, for beamforming like spatial resource allocation methods. We note that the work in [11] is closest to our work in spirit with one major difference. In [11], authors consider the impact of using $L$-rounds of ARQ in a MIMO channel, and show that additional delay changes the diversity-multiplexing behaviour in much the same way as our results. Each ARQ round provides one-bit information about the channel output and hence $L$-rounds provide $L$ bits of information. In contrast, we consider impact of channel information, not the channel output in this paper. In our opinion, the two works are complementary and could form the basis of answering a more fundamental question. If we can send only $b$-bits of feedback per unit time, then how should those $b$ bits be selected. The obvious options include quantizing the channel and channel output (equivalently decoder state). The difference between the two possibilities is that channel feedback occurs before the data transmission and channel output feedback after the data transmission. A priori it is unclear which form of feedback provides the maximal gain.

We further note that work of $[7,12]$ also identified that additional queuing delay can improve the outage performance. The change of slope of the outage as a function of SNR was observed, though no results regarding actual increase in diversity level were proven. In this paper, we show that queuing delay does increase diversity for all multiplexing gains by simultaneously using rate and power control.

The fact that diversity order of the system can be increased beyond the available degrees of freedom is rather counter-intuitive. Part of the elegance of the results in [8] is that the diversitymultiplexing tradeoff curve completely matches the intuition behind degrees of freedom framework, where each additional d.o.f. can be used to increase either throughput or diversity. The degrees of freedom framework is a statistical notion, where the degrees are related to the probability distribution of the channel. When the transmitter has no channel state information, it has to use the same action for each state and has no opportunity for adaptation.

However, with channel state information, the transmitter has the opportunity to adapt to current channel conditions. Thus, the transmitter can adapt how it uses the instantaneous d.o.f. This per 
state adaptation is key to changing the probability of outage events, saving power in the good states to put more power in the poor channel states and thus increasing the number of states in which a successful transmission occurs.

The rest of the paper is organized as follows. In Section 2, we introduce our channel and feedback model. In Section 3, we provide the results pertaining to the case of perfect channel information at the transmitter, while Section 4 and Section 5 have the quantized power control and diversitymultiplexing tradeoff bounds, respectively, for finite bit feedback model. Finally, a joint rate and power extension is studied in Section 6, where we also discuss the system design implications of our proposed power control method. We conclude in Section 7.

\section{Problem Formulation}

In this section, we first describe the channel and feedback system model, followed by the optimization objective of feedback design.

\subsection{Channel Model}

We consider a multiple antenna channel with $M$ transmit and $N$ receive antennas (Figure 1). When the channel coherence time is larger than the duration of transmission of a codeword, channel can be modeled as a block fading channel with the following input-output relation

$$
Y=H S+W
$$

In $1, S$ is $M \times 1$ transmitted vector, $H$ is the $N \times M$ channel matrix, and $W$ is the $N \times 1$ additive white Gaussian noise (AWGN). Channel matrix is assumed to have entries $h_{i j}$, that are scalar channel coefficients between the $j^{\text {th }}$ transmit and $i^{t h}$ receive antenna. Channel coefficients are assumed to be independent complex random variables with circularly symmetric Gaussian distribution with zero mean and unit variance, $\left[h_{i j}\right] \in \mathcal{C N}^{N \times M}$. The input signal vector $S \sim \mathcal{C N}^{M}\left(0, \Sigma_{S}\right)$ and the additive noise $W \sim \mathcal{C N}^{N}\left(0, I_{N}\right) .{ }^{1}$ Finally, the transmitter is equipped with a finite average power $P_{a v}$ such that $\mathbb{E}\left[S^{\dagger} S\right] \leq P_{a v}$.

\subsection{System Model}

The complete feedback system is depicted in Figure 1, where there is fast feedback link between the transmitter and receiver. The receiver is assumed to have perfect knowledge of the MIMO channel $H$, and sends a feedback codeword $Q(H)$ to the transmitter. For the case of perfect information at transmitter $Q(H)=H$ and for finite rate feedback $Q(H): \mathbb{C}^{M N} \rightarrow\{1, \ldots, L\}$. On receiving the feedback codeword $Q(H)$, the transmitter uses the channel information to adapt its transmission scheme for actual transmission of the data. We assume that the feedback is error-free and delayfree. In our recent work, we have also analyzed the impact of feedback errors for a two-way training model [13].

The finite bits of feedback about the channel can be used to perform either beamforming [4-6], power control $[14,15]$ or rate control $[16,17]$ among other possible transmission adaptations. In this paper, we will focus mainly on temporal power control (with a discussion of rate control in

\footnotetext{
${ }^{1} I_{t}$ is the $t \times t$ identity matrix.
} 
Section 6). In temporal power control, all transmit antennas are used simultaneously with the same power $P(Q(H))$ or more concisely $P_{Q}(H)$. In this case, the transmitted signal $S=P_{Q}(H)^{1 / 2} X$ and the received signal can be written as

$$
Y=H P_{Q}(H)^{1 / 2} X+W .
$$

Note that $X$ has a unit power, i.e., $\operatorname{tr}\left\{\mathbb{E}\left[X X^{\dagger}\right]\right\}=1$. If $X$ has iid components, then $\mathbb{E}\left[X X^{\dagger}\right]=$ $I_{M} / M$. The only constraint imposed is on the long term average power constraint of the transmitted signal $P(Q(H))^{1 / 2} X$. That is,

$$
\left.\lim _{T \rightarrow \infty} \frac{1}{T} \sum_{i=1}^{T}\left[P_{Q}\left(H_{i}\right)^{1 / 2} X_{i}\right)\right]^{\dagger} P_{Q}\left(H_{i}\right)^{1 / 2} X_{i} \leq P_{a v}
$$

where $H_{i}$ and $X_{i}$ represent the channel and the input signal at time $i$, respectively. Assuming a stationary and ergodic channel and applying the law of large numbers, we can replace the time average with the ensemble average, i.e.,

$$
\begin{aligned}
\left.\lim _{T \rightarrow \infty} \frac{1}{T} \sum_{i=1}^{T}\left[P_{Q}\left(H_{i}\right)^{1 / 2} X_{i}\right)\right]^{\dagger} P_{Q}\left(H_{i}\right)^{1 / 2} X_{i} & =\mathbb{E}\left[X^{\dagger} P_{Q}(H) X\right] \\
& \stackrel{a}{=} \mathbb{E}\left[\operatorname{tr}\left\{P_{Q}(H) X X^{\dagger}\right\}\right] \\
& \stackrel{b}{=} \frac{1}{M} \operatorname{tr}\left\{\mathbb{E}\left[P_{Q}(H)\right]\right\},
\end{aligned}
$$

where (a) is obtained by applying $x^{\dagger} y=\operatorname{tr}\left\{y x^{\dagger}\right\}, \forall x, y$ in a vector space $V$, and (b) by having input signal with unit energy, i.e., $\mathbb{E}\left[X X^{\dagger}\right]=\frac{1}{M} I_{M}$. The expectation in (4) is taken with respect to the distribution of $H$.

\subsection{Objective}

Our optimization objective is to attain maximum diversity order $d$ for a fixed multiplexing gain of $r$. The optimum power allocation is known for perfect CSIT, therefore, the optimal diversitymultiplexing curve can be determined. However, the optimal power control with finite feedback is not known, thus our result is a lower bound for the optimal diversity-multiplexing tradeoff. The diversity order of any transmission scheme is defined as the slope at which the probability of outage decays as the function of SNR or equivalently transmit power $P_{a v}$. More formally, it is defined as [8]

$$
d=\lim _{P_{a v} \rightarrow \infty}-\frac{\log \left(\Pi\left(P_{a v}, R\left(P_{a v}\right)\right)\right)}{\log \left(P_{a v}\right)} .
$$

where $\Pi\left(P_{a v}, R\left(P_{a v}\right)\right)$ is the probability of outage with power $P_{a v}$ and rate $R\left(P_{a v}\right)$. Note that outage is typically understood as an event in which the transmitter sends a codeword and receiver is unable to decode it correctly [18]. However, with channel state information, the transmitter may choose not to send any codeword in poor channel conditions. In the conventional definition, such an action implies no outage, leading to potentially no outage system. We believe for feedback based system, a more general definition is necessary. We visualize the system as a time slotted system, in which each slot can accommodate a full codeword and the channel state is constant over the whole time-slot. 
The outage event is then defined as lack of information at the receiver at end of each time-slot. Thus the outage event can occur in two possible ways - either the transmitter sends no information or the transmission is in error. In either case, the receiver gets no useful information. We note that this definition is useful when the system is not allowed to queue the packets and each packet is either sent or dropped. If queuing is allowed at the transmitter, then the above outage definition still applies as either a packet drop by the transmitter or erroneous decoding at the receiver [12].

The complementary metric of multiplexing gain is defined as the rate at which throughput scales as a function of $\log \left(P_{a v}\right)$, computed as

$$
r=\lim _{P_{a v} \rightarrow \infty} \frac{R\left(P_{a v}\right)}{\log \left(P_{a v}\right)} .
$$

We note that multiplexing gain, $r$, can not be larger than the rate of growth of ergodic capacity, $m=\min (M, N)$ with any amount of channel information at either transmitter or receiver.

\section{Perfect Transmitter and Receiver Information}

In this section, we will derive the diversity-multiplexing tradeoff curve for multiple antenna channels with perfect channel information at both the transmitter and receiver (CSITR). A complementary analysis with only receiver information was first derived in [8]. It is apparent that receiver only analysis is a lower bound to our finite bit feedback analysis in the next section, and the perfect CSITR analysis in this section yields an upper bound. The transmitter channel information is predominantly employed to perform power control, rate control and/or transmitter beamforming. In this section, we will restrict our attention to methods which no not perform rate control, and always send at a constant rate.

Both power control and beamforming are examples of adaptive power allocation. In the case of beamforming, only spatial power adaptation is performed while each codeblock receives the same total power and thus there is no temporal adaptation of the total power given to the codewords. In this section, we will compare the asymptotic performance of three methods. The first is the optimal power control which performs a joint temporal and spatial power control, and was derived in $[3,19]$. Thus, optimal power control implicitly performs beamforming. The second is purely temporal power control with equal power allocation on each spatial direction. Temporal power control does not require information about eigen-directions and thus needs lesser feedback about the channel state information than optimal power control. Finally, we will consider spatial power control, equivalently beamforming, and thus compare the efficacy of temporal versus spatial opportunism.

First, we state the diversity-multiplexing tradeoff with no transmitter information and perfect receiver information as derived in [8].

Theorem 1 (Theorem 2, [8]) Assume that the codeword length $l>m+n-1$. The optimal diversity-multiplexing tradeoff curve $d^{*}(r)$ is given by the piecewise linear function connecting the points $\left(r, d^{*}(r)\right), k=0,1, m$ where

$$
d^{*}(r)=(m-r)(n-r)
$$

Recall $n=\max (M, N)$ and $m=\min (M, N)$. 
The diversity multiplexing curve captures the intuitive tradeoff in using the spatial degrees of freedom to increase data rates or reliability or a combination of the two objectives. Without any instantaneous channel information at the transmitter, the transmitter cannot perform any rate, spatial or temporal power control and uses all its power in all spatial directions for all the channel conditions. In the next three theorems, we will explore the efficacy of power control and compare it with Theorem 1.

Theorem 2 (Optimal Power Control) With the perfect channel information at both transmitter and receiver, the diversity order $d^{\dagger}(r)$ of optimal power control for multiplexing gain $r$ is given as

$$
d^{\dagger}(r)=\left\{\begin{array}{ll}
\infty & r \in[0, m) \\
\infty & r=m \text { and } n>2 m .
\end{array} .\right.
$$

Proof: See Appendix B.

The Theorem 2 does not include the case when $m \leq n \leq 2 m$ and $r=m$. We conjecture that when $r=m=n$ the diversity order is zero. The intuition is based on the optimum power control in [3] is which the optimal power control depends on the geometric mean of eigenvalues. Whether the channel parameter is the geometric mean of eigenvalues of any additive or multiplicative function of eigenvalues, in either case the distribution of the parameter is of form $f_{\eta}(x)=\kappa x^{n-m} e^{-x} q(x)$, 2 where $\kappa$ is a normalizing constant and $q(x)$ is a polynomial such that $q(0) \neq 0$. Therefore, for $n=m$, the average power constraint $\int_{\gamma_{0}}^{\infty} 1 / x f_{\eta}(x) d x=1$ has a singularity at $x=0$. That means that $\gamma_{0} \nrightarrow 0$. In fact $\gamma_{0}=c$ for some constant $c$, which in turn implies that the outage probability, $\operatorname{Pr}\left\{\eta<\gamma_{0}\right\}$ is constant. Similarly, when $n>m$, the channel inversion resolved by roots of channel distribution at origin results in outage free power allocation for all channel conditions. Thus, the outage probability is zero and diversity order is infinite.

When $r \neq m$ but $n=m, \gamma_{0}$ is still a constant but a function of $P_{a v}$ such that as $P_{a v}$ approaches infinity, $\gamma_{0}$ goes to zero. Thus, a non-zero diversity order is achievable. On the other hand, when $n>m$ the distribution of the channel parameter has a root at the origin, that is, $f_{\eta}(0)=0$, which cancels out the singularity of the channel inversion. Therefore, it is possible to make $\gamma_{0}$ small and yet not violate the average power constraint. The challenge at $r=m$ is that the equation for average power constraint is independent of $P_{a v}$ and hence $\gamma_{0}$ is a constant. If this constant is zero, then the diversity order is infinity, but if the constant is non-zero, then the outage is constant and the diversity order is zero. The complete solution of $\gamma_{0}$ depends on the constant $\kappa$ and the polynomial $q(x)$, which make the analysis involved.

In general the behavior of systems at highest multiplexing gain $r=m$ is hardest to predict. The rate is growing with SNR as fast as the ergodic capacity does, while the block length is kept fixed. Next theorem shows that when CSI is only available at the receiver, the diversity order at $\mathrm{r}=\mathrm{m}$ is not achievable.

Theorem 3 At highest multiplexing gain $r=m$, the outage probability $\Pi_{C S I R} \nrightarrow 0$ as $P_{a v} \rightarrow \infty$. Thus, $r-m$ is not achievable.

\footnotetext{
${ }^{2}$ It is not relevant to the discussion in this paper, but the distribution of $\bar{\lambda}=\left(\prod \lambda_{i}\right)^{1 / m}$ can be found by classical method of finding the distribution of $z=\log \bar{\lambda}$ and it can be shown that the roots of the distribution at origin has the multiplicity of $(n-m)$. Similarly through the convolution argument it can be shown that the distribution of the summation of arbitrary number of eigenvalues including the smallest has roots at origin with multiplicity of no larger than $(n-m)$.
} 
Proof: See appendix A.

A comparison of Theorems 1 and 2 shows that the transmitter information completely changes the asymptotic decay rate of probability of outage from a finite decay in perfect CSIR system to exponential rate of decay in perfect CSIRT system. The change in decay rate can be completely attributed to optimum power control, which was derived in [19] and stated below with transmission rate as $r \log (\mathrm{SNR})$

$$
P_{i}=\left[\left(\frac{2^{\frac{r \log \mathrm{SNR}}{m}}}{\left(\prod_{j=1}^{m} \lambda_{j}\right)^{\frac{1}{m}}}\right)-\frac{1}{\lambda_{i}}\right]^{+} .
$$

The power $P_{i}$ is the power allocated to the eigenvector corresponding to eigenvalue $\lambda_{i}$. Thus, not only there is spatial power control, the total power allocated for a codeword depends on the current channel $H$ leading to temporal power control. In the next theorem, we allocate equal power to each antenna (no spatial power control) which can change from one time-slot to another based on current channel conditions, i.e., temporal power control.

Theorem 4 (Temporal Power Control) When temporal power control is performed with same power on all antennas, then the diversity order $d_{t}(r)$ is given as

$$
d_{t}(r)= \begin{cases}\infty & r<m \\ 0 & r=m, n<2 m \\ \infty & r=m, n \geq 2 m\end{cases}
$$

Proof: Consider a power allocation policy based on the smallest eigenvalue $\lambda_{m}$. It is well known that the distribution of the smallest eigenvalue is given by [20]

$$
f_{\lambda_{m}}(x)=\frac{1}{\Gamma(n-m+1)} x^{n-m} e^{-x},
$$

The outage minimizing power allocation [3] is of the form

$$
P\left(\lambda_{m}\right)= \begin{cases}\frac{2^{R}-1}{\lambda_{m}}, & \lambda_{m}>\gamma_{0} \\ 0, & \text { otherwise }\end{cases}
$$

where $\gamma_{0}$ is the boundary of the outage region and is obtained from the relation for average power constraint, i.e.,

$$
\int_{\gamma_{0}}^{\infty} m P(x) f_{\lambda_{m}}(x) d x \leq P_{a v}
$$

Then, the information outage occurs only when there is no transmission,

$$
\Pi_{E P}=\operatorname{Pr}\left\{\lambda_{m}<\gamma_{0}\right\} .
$$

Replacing the distribution in (13) and solving for $\gamma_{0}$ we have

$$
\int_{\gamma_{0}}^{\infty} m \frac{2^{\frac{r}{m} \log P_{a v}}-1}{\Gamma(n-m+1) x} x^{n-m} e^{-x} d x \leq P_{a v}
$$


which can be simplified to

$$
\int_{\gamma_{0}}^{\infty} x^{n-m-1} e^{-x} d x \leq \frac{\Gamma(n-m+1)}{m} P_{a v}^{1-r / m}
$$

For $r<m$, the right hand side of (16) goes to infinity as $P_{a v}$ goes to infinity, whereas the left hand side of (16) is bounded above by $\Gamma(n-m)$ when $\gamma_{0}=0$. Therefore, for $P_{a v}>\Gamma(n-m) * m / \Gamma(n-m+1)$, the cutoff threshold $\gamma_{0}=0$ and the outage probability $\operatorname{Pr}\left\{\lambda_{m}<\gamma_{0}\right\}=0$, which results in an infinite diversity order.

For $r=m$, the right hand side of Equation (16) is independent of $P_{a v}$. Therefore, in order to have diversity order of infinity, we need to have zero outage probability, which is equivalent of $\gamma_{0}=0$. For $\gamma_{0}=0$ inequality (16) becomes

$$
\begin{aligned}
1 & \leq \frac{\Gamma(n-m+1)}{m \cdot \Gamma(n-m)} \\
& =\frac{n-m}{m},
\end{aligned}
$$

or equivalently $n \geq 2 m$. Therefore, for $n \geq 2 m$ the outage probability is zero and diversity order of infinity is achieved at $r=m$. However, for $n<2 m, \gamma_{0}=0$ can not be the solution to (13) and therefore the outage would be non-zero. ${ }^{3}$

Thus, the asymptotic behavior for all but maximum multiplexing gain is unchanged. In some cases (such as $n=m$ ), for the largest multiplexing gain, the diversity order is reduced from $\infty$ to zero, much like in a system with no transmitter information. The reason for such dramatic change is insistence on equal power allocation in all eigenvalue directions. In this case, the diversity order at maximum multiplexing gain is determined by the minimum eigenvalue which leads to a zero diversity gain.

Theorem 5 (Spatial Power Control a.k.a. Beamforming) When only spatial power control is performed with same total power for all channel realizations, then the diversity order $d_{s}(r)$ is same as the system with no transmitter information [8], and is given by a piecewise linear function between the following points $\left(r, d_{s}(r)\right)$ for $r=0,1, \ldots, m$ where

$$
d_{s}(r)=(m-r)(n-r)=d^{*}(r) .
$$

Proof: The advantage of beamforming at the transmitter is in that signals at the receiver can be combined coherently and hence the total received SNR is improved. The expression for mutual information is given by

$$
I(X ; Y \mid H)=\log \left(\operatorname{det} I_{N}+P_{a v} H H^{\dagger}\right) .
$$

It is shown in [21], that all systems in which the expression for mutual information is of form $\log \operatorname{det}\left(I+\alpha P_{a v} H H^{\dagger}\right)$, have the same diversity order. For a system with beamforming at the transmitter $\alpha=1$, and for a channel model with CSI only at the receiver $\alpha=1 / M$. Therefore, beamforming does not change the diversity order.

\footnotetext{
${ }^{3}$ Note that the optimum choice of feedback is an open problem, and a better choice of feedback may change the statement of the theorem.
} 
The above theorem demonstrates that beamforming alone, with no temporal power control, leads to no improvement in asymptotic rates. Thus, while beamforming leads to SNR improvement in outage by coherent combining of the received signals, it has no impact on rate of decay of outage probability with SNR [21]. The above sequence of results demonstrate that temporal power control is better use of channel information than spatial power control. In other words, if there were only a finite bits of information available at the transmitter about the channel, there impact may be highest if all of them are allocated to performing temporal power control.

Example 1: Consider the case of single-antenna system with $n=m=1$. With perfect information about the channel, the transmitter action includes a truncated power control and a phase correction at the transmitter. Thus, the received signal is

$$
y=\left\{\begin{array}{ll}
\left(e^{r \log \mathrm{SNR}}-1\right) s+w, & |h|^{2} \geq \gamma_{0} \\
w, & |h|^{2}<\gamma_{0}
\end{array} .\right.
$$

The threshold $\gamma_{0}$ is chosen to meet the average power constraint. Note that since $\mathbb{E}\left[1 /|h|^{2}\right]=\infty$, then in order to have a finite power constraint $\gamma_{0}>0$, for all SNR. The outage probability is approximately $\Pi \approx \exp \left(-\mathrm{SNR}^{1-r}\right)$ for $r \in[0,1)$, which decays exponentially fast like in Gaussian channels. Thus the diversity order is infinite for all multiplexing gains less than the maximum.

Example 2: In a $1 \times 2$ system the channel distribution is slightly different. The channel norm $\|h\|^{2}$ distribution (with maximum ration combining at the receiver) is $f_{\|h\|^{2}}(x)=x e^{-x}$. Since, $f_{\|h\|^{2}}(0)=0$, then $\mathbb{E}\left[1 /\|h\|^{2}\right] \leq c$ for some constant $c$. Thus, for $P_{a v}>c$ it is possible to invert all channel conditions and yet meet the average power constraint. Therefore, for all multiplexing gain $r \in[0,1]$, a zero outage and equivalently infinite diversity order is achievable. Note that unlike single antenna case (Example 1), diversity order of infinity is achieved at $r=1$.

For the rest of the paper, we will largely focus our attention on finite feedback based temporal power allocation since it achieves large gains without the need for learning the eigenvectors of the channels (needed by optimal and spatial power control methods).

\section{Quantized Power Control}

In this section, we will develop a suboptimal finite bit quantizer to perform temporal power control. The quantizer has a simple form which allows recursive calculation of all quantization thresholds, and simplifies the subsequent analysis of diversity-multiplexing tradeoff.

\subsection{Preliminaries}

In temporal power control, each eigen-direction receives the same power which varies from codeword to codeword based on current channel conditions. In this case, the mutual information using a full-rank Gaussian space-time code with covariance $\frac{1}{M} I_{M}$ and power $P(H)$ is given by

$$
I(S ; Y \mid Q(H))=\log \operatorname{det}\left(I_{N}+\frac{P(H)}{M} H I_{M} H^{\dagger}\right),
$$


where $I_{M}$ is $M \times M$ identity matrix and $H^{\dagger}$ is the Hermitian conjugate of $H$. The power $P(H)$ is the equal power assigned to all the eigenvectors and depends on channel conditions $H$. The mutual information in Equation (19) can be rewritten as

$$
\begin{aligned}
I(S ; Y \mid Q(H)) & =\log \operatorname{det}\left(I_{N}+\frac{P(H)}{M} H H^{\dagger}\right), \\
& \stackrel{(a)}{=} \log \operatorname{det}\left(I_{N}+\frac{P(H)}{M}\left(U \Lambda^{1 / 2} V^{\dagger}\right)\left(U \Lambda^{1 / 2} V^{\dagger}\right)^{\dagger}\right), \\
& =\log \operatorname{det}\left(I_{m}+\frac{P(H)}{M} \Lambda\right), \\
& =\sum_{i=1}^{m} \log \left(1+\frac{P(H)}{M} \lambda_{i}\right),
\end{aligned}
$$

where (a) is obtained by replacing $H$ with its singular value decomposition, $H=U \Lambda^{1 / 2} V^{\dagger}$ and $m=$ $\min (M, N)$. Furthermore, $\left\{\lambda_{i}\right\}_{i=1}^{m}$ are the non-zero eigenvalues of the matrix $H H^{\dagger}$. Thus for temporal control, the power $P(H)$ is a mapping from the $m$-dimensional eigenvector space $\left[\begin{array}{llll}\lambda_{1} & \lambda_{2} & \cdots & \lambda_{m}\end{array}\right]$ to non-negative real space $\mathbb{R}^{+} \cup\{0\}$, and is a complex non-linear vector quantization problem.

Instead of using a vector quantizer, we will further simplify the quantization problem by focusing on only one of the $m$ eigenvalues. The power $P(H)$ is then determined by a single eigenvalue $\lambda_{i}$ and the functional relationship will be denoted by $P\left(\lambda_{i}\right)$ whenever needed. The simplification reduces the $m$-dimensional vector quantization problem to a single dimensional vector quantization. Next we consider the design of optimal scalar quantizer for a fixed eigenvalue.

\subsection{Optimal Single Eigenvalue Quantizer}

A typical continuous and quantized power control is illustrated in Figure 2. The continuous curve in Figure 2 corresponds to channel inversion power allocation, and the piecewise continuous step function is its quantized approximation with 5 quantization bins. The $x$-axis in Figure 2 represents one of the non-zero eigenvalues of the $H^{\dagger}$ matrix, say $\lambda_{i}$. If the receiver observes that the eigenvalue $\lambda_{i}$ lies in the interval $\left[\gamma_{2}, \gamma_{3}\right)$, the index of this interval is fedback to the transmitter. Transmitter in turn allocates power $P_{2}=k / \gamma_{2}, k=\left(2^{R\left(P_{a v}\right)}-1\right)$. Since the transmitter does not know the exact value of the channel, it should allocate power based on the worst case scenario such that for all channel conditions in that interval, an outage free communication is guaranteed.

The channel quantizer is described by $L$ quantization thresholds $\left\{\gamma_{1}, \gamma_{2}, \ldots, \gamma_{L-1}\right\}$ which define $L$ quantization bins $\left\{\left[0, \gamma_{1}\right),\left[\gamma_{1}, \gamma_{2}\right), \ldots,\left[\gamma_{L-1}, \infty\right)\right\}$, where $L=2^{B}$. For each quantization bin $\left[\gamma_{j}, \gamma_{j+1}\right), j \geq 1$, power $P_{j}=k / \gamma_{j}$ with $k=\left(2^{R\left(P_{a v}\right)}-1\right)$. Since origin belongs to the first quantization bin, a finite power $P_{0}$ is assigned to this bin such that the total average power constraint is satisfied. Note that $0<\left(\gamma_{0}=k / P_{0}<\gamma_{1}\right.$ such that power $P_{0}$ only guarantees outage free communication for $\lambda_{i} \geq \gamma_{0}$. For $\lambda_{i}<\gamma_{0}$, the power $P_{0}$ is not sufficient to prevent outage and thus the probability of outage is given by

$$
\Pi\left(R\left(P_{a v}\right)\right)=\operatorname{Prob}\left\{\lambda_{i}<\gamma_{0}\right\} .
$$

First we note that the average power constraint of (4) with quantized power levels is reduced to

$$
\mathbb{E}\left[P^{*}\left(Q^{*}\left(\lambda_{i}\right)\right)\right]=P_{0} F_{\lambda_{i}}\left(0, \gamma_{1}\right)+\cdots+P_{L-1} F_{\lambda_{i}}\left(\gamma_{L-1}, \infty\right)
$$


where $F_{\lambda_{i}}(\alpha, \beta)=\int_{\alpha}^{\beta} f_{\lambda_{i}}(x) d x$ is the probability mass concentrated in the interval $[\alpha, \beta]$ and $f_{\lambda_{i}}(\cdot)$ is the probability distribution of $\lambda_{i}$.

Then the optimum channel quantizer $Q^{*}$ along with the optimum quantized power allocation $P^{*}\left(Q^{*}\right)$ are solutions to the outage minimization problem

$$
\left\{P^{*}\left(Q^{*}\left(\lambda_{i}\right)\right), Q^{*}\left(\lambda_{i}\right)\right\}=\arg \min _{\mathbb{E}\left(P\left(Q\left(\lambda_{i}\right)\right)\right) \leq P_{a v}} \Pi\left(R\left(P_{a v}\right)\right) .
$$

In $[3,19]$ authors showed that the problem (23) has a dual which can be expressed by

$$
\left\{P^{*}\left(Q^{*}\left(\lambda_{i}\right)\right), \mathcal{Q}^{*}\left(\lambda_{i}\right)\right\}=\arg \min _{\Pi\left(R\left(P_{a v}\right)\right) \leq \alpha} \mathbb{E}\left(P\left(Q\left(\lambda_{i}\right)\right)\right) .
$$

The constraint on outage in dual problem of (24) is the same as saying $\operatorname{Pr}\left\{\lambda_{i}<\gamma_{0}\right\} \leq \alpha$, which can be solved for $\gamma_{0}$. Knowing $\gamma_{0}$, power level $P_{0}=k / \gamma_{0}$ is known. Therefore the dual problem (24) is reduced to an unconstrained optimization problem in a space with one less dimension than the original problem. The solution to the reduced optimization problem must satisfy the first order KKT condition, $\vec{\nabla}_{P\left(\lambda_{i}\right)} \mathbb{E}_{\lambda_{i}}\left[P\left(\lambda_{i}\right)\right]=0$, which leads to the following system of nonlinear equations

$$
\begin{cases}\frac{f_{\lambda_{i}}\left(\gamma_{1}\right)}{\gamma_{0}}-\frac{F_{\lambda_{i}}\left(\gamma_{1}, \gamma_{2}\right)}{\gamma_{1}^{2}}-\frac{f_{\lambda_{i}}\left(\gamma_{1}\right)}{\gamma_{1}} & =0 \\ \frac{f_{\lambda_{i}}\left(\gamma_{2}\right)}{\gamma_{1}}-\frac{F_{\lambda_{i}}\left(\gamma_{2}, \gamma_{3}\right)}{\gamma_{2}^{2}}-\frac{f_{\lambda_{i}}\left(\gamma_{2}\right)}{\gamma_{2}} & =0 \\ \vdots & \vdots \\ \frac{f_{\lambda_{i}}\left(\gamma_{L-1}\right)}{\gamma_{L-2}}-\frac{F_{\lambda_{i}}\left(\gamma_{L-1}, \infty\right)}{\gamma_{L-1}^{2}}-\frac{f_{\lambda_{i}}\left(\gamma_{L-1}\right)}{\gamma_{L-1}} & =0 .\end{cases}
$$

The solution to system of equations in (25) does not admit a closed form and includes nonlinear transcendental equations for Rayleigh channels. In next section we find a suboptimum channel quantizer which allocates equal total power to each quantization bin, i.e., the product of power level and probability mass is equal across all quantization bins.

\subsection{Equi-Power Quantization}

Consider the $j$ th equation in $(25)\left(1 \leq j \leq L-1\right.$, with $\left.\gamma_{L}=\infty\right)$, that is,

$$
\frac{1}{\gamma_{j-1}} f_{\lambda_{i}}\left(\gamma_{j}\right)-\frac{1}{\gamma_{j}^{2}} F_{\lambda_{i}}\left(\gamma_{j}, \gamma_{j+1}\right)-\frac{1}{\gamma_{j}} f_{\lambda_{i}}\left(\gamma_{j}\right)=0
$$

We can rewrite (26) as,

$$
\begin{aligned}
\frac{1}{\gamma_{j}}\left(\gamma_{j+1}-\gamma_{j}\right) f_{\lambda_{i}}\left(\gamma_{j}\right) & =\frac{1}{\gamma_{j+1}} F_{\lambda_{i}}\left(\gamma_{j+1}, \gamma_{j+2}\right) \\
P_{j}\left(\gamma_{j+1}-\gamma_{j}\right) f_{\lambda_{i}}\left(\gamma_{j}\right) & =P_{j+1} F_{\lambda_{i}}\left(\gamma_{j+1}, \gamma_{j+2}\right) .
\end{aligned}
$$

As number of bits in feedback, $B=\log _{2}(L)$, approaches infinity, the length of quantization bins, $\left(\gamma_{j}, \gamma_{j+1}\right)$, approaches zero, and hence by mean value theorem [22], we can further simplify (27) when $B \rightarrow \infty$ as

$$
P_{j} F_{\lambda_{i}}\left(\gamma_{j}, \gamma_{j+1}\right) \approx P_{j+1} F_{\lambda_{i}}\left(\gamma_{j+1}, \gamma_{j+2}\right)
$$


The term $P_{i} F_{\lambda_{i}}\left(\gamma_{j}, \gamma_{j+1}\right)$ is the total allocated power to the $j$ th bin. Thus from (28), it follows that an approximation to the optimal power allocation is to allocate equal total power to each quantization bin. From the above discussion, it also follows that the equal allocation power control is asymptotically (in number of quantization bins $L$ ) approaches the optimum quantized power. The above approximate solution (28) can now be used in the primal problem (23). Authors in [3] showed that the solution to $(23)$ is on the boundary of constraint set, i.e., at the optimum point, $P^{*}\left(Q^{*}\left(\lambda_{i}\right)\right)$, we have $\mathbb{E}\left[P^{*}\left(Q^{*}\left(\lambda_{i}\right)\right)\right]=P_{a v}$. More precisely, at $P^{*}\left(Q^{*}\left(\lambda_{i}\right)\right)$ we have

$$
P_{0} F_{\lambda_{i}}\left(0, \gamma_{1}^{*}\right)+\cdots+P_{L-1} F_{\lambda_{i}}\left(\gamma_{L-1}^{*}, \infty\right)=P_{a v} .
$$

Combining (29) with (28) we get,

$$
P_{j} F_{\lambda_{i}}\left(\gamma_{j}^{*}, \gamma_{j+1}^{*}\right)=\frac{P_{a v}}{L}, \quad \forall j \in\{0,2, \ldots, L-1\},
$$

with $\gamma_{L}^{*}=\infty$ and $\gamma_{0}^{*}=0 .{ }^{4}$ For $j=(L-1)$ in (30) we have

$$
P_{L-1} F_{\lambda_{i}}\left(\gamma_{L-1}^{*}, \infty\right)=\frac{P_{a v}}{L} .
$$

Also by channel inversion power allocation we have $\gamma_{L-1}^{*}=k / P_{L-1}$. Hence identity (31) is only a function of $P_{L-1}\left(\right.$ or $\left.\gamma_{L-1}^{*}\right)$ and we can solve (31) for $P_{L-1}\left(\right.$ or $\gamma_{L-1}^{*}$ ). Replacing the value for $\gamma_{L-1}^{*}$ in (30) for $j=(L-2)$, we end up with an equation with a single variable $P_{L-2}$ (and corresponding threshold $\gamma_{L-2}^{*}$ ). By recursively repeating the same procedure, we can obtain all the power levels, $\left\{P_{j}\right\}_{j=1}^{L}$.

Figure 3 compares the performances of systems with quantized feedback, optimal, and equal allocation power control, and a system with perfect CSI at both ends, as a function of SNR. A single transmit and single receive antenna system is considered, and the feedback rate is $B=\log _{2}(3)$ bits/codeblock. Note that the performance of optimal and equi-power schemes are not distinguishable in Figure 3 indicating that equal power allocation performs very close to optimum for the range of simulated SNRs.

Note that as $P_{a v}$ approaches infinity all the quantization thresholds $\gamma_{i}$ 's, approach to zero. Therefore, the length of all the quantization bins, $\left(\gamma_{i}, \gamma_{i+1}\right]$ approaches zero, which satisfies the mean value theorem condition applied to (27). Thus, the suboptimum quantizer found in this section is asymptotically optimum as $P_{a v}$ approaches infinity, and the diversity order analysis based on the suboptimum power control would be the same with that of the optimum power control based on the optimum quantizer.

The derivation of the quantization thresholds in this section is independent of the distribution of the channel parameter, as long as the distribution is continuous and differentiable. In the next section we use the tools developed in here to characterize the outage performance and quantify the diversity order of channel with Rayleigh distribution and finite rate feedback.

\section{Diversity-Multiplexing Tradeoff with Quantized Feedback}

The main idea in deriving the diversity-multiplexing tradeoff bound is as follows. To achieve a multiplexing gain of $i$, we will use the largest $i$ eigenvalues and ensure that the allocated power is

\footnotetext{
${ }^{4}$ to prevent more complication and only in here, we abuse the notation $\gamma_{0}=0$ to indicate the left boundary of the left most quantization bin and every where else by $\gamma_{0}$ we mean the threshold that determines the outage boundary and is equal to $k / P_{0}$
} 
sufficient to achieve minimal outage. To do that we will quantize $\lambda_{i}$ using the equi-power quantizer developed in Section 4.3, which is designed to closely approximate the optimal single-eigenvalue quantizer of Section 4.2. Having chosen the quantization mechanism based on single eigenvalue, we then can study scalar eigenvalue distribution for high SNR regime which requires characterizing the eigenvalue distribution around zero.

Theorem 6 (Diversity-Multiplexing Tradeoff) In a MIMO system, for a multiplexing gain $r$, when a scalar quantizer with $L$ bins is used at the receiver the diversity order is given by

$$
d=\max _{i \in\{j, \ldots, m\}}\{(1-\alpha)(n-j+1)(m-j+1) G(m, n, i, L)\}
$$

where $j=\lceil r\rceil, \alpha=r / i$, and $i$ is the argument that maximizes (32). Further the function $G(m, n, i, L)$ is defined as

$$
G(m, n, i, L) \triangleq \sum_{l=0}^{L-1}[(n-i+1)(m-i+1)]^{l} .
$$

Proof: See Section 5.1.

Example 3: Consider the case of single transmit and receive antenna system, $m=n=1$. The diversity order for zero multiplexing gain $(r=0, i=j=1)$ is given by $d=2^{B}$ (recall $L=2^{B}$ ). In contrast, with no CSIT, the diversity order is one while with perfect CSIT, the diversity order is $\infty$. Thus, as expected, partial CSIT results in a finite diversity order which increases with the number of bits. In other words, feedback adds to the diversity order of the system, primarily by temporally utilizing spatial degrees of freedom. The more interesting aspect is the rate at which the diversity order grows; the next example clarifies that further.

Example 4: Consider the case of $m \times n$ system with $B$ bits of feedback. The diversity order for zero multiplexing gain is $d=m n\left((m n)^{2^{B}}-1\right) /(m n-1)$. The above expression clearly shows that the diversity order is doubly exponential in number of feedback bits $B$.

It is interesting to notice that for a SISO system with $B$ bits of feedback $\left(L=2^{B}\right)$ and $r=0$, the diversity order is $d_{L}=L$ which is larger than diversity order with CSIR only, $d_{C S I R}=1$. In fact it has a diversity order of a MISO/SIMO system with $L$ antennas and CSI at receiver, which suggests that spatial diversity in CSIR systems can be replaced by feedback diversity in a feedback based system.

The diversity order in the statement of the Theorem 6 consists of three parts; (i) the maximization, (ii) the diversity order factor $(1-\alpha)(n-j+1)(m-j+1)$, (iii) and the effect of the quantization $G(m, n, i, L)$. The maximization is simply finds the largest achievable diversity order, given the desired multiplexing gain. The factor $(1-\alpha, \alpha=r / i$ is the result of dividing the desired rate equally among the at most $i=\lceil r\rceil$ many "parallel" channels. Therefore, $i$ indicates the smallest integer larger than the desired multiplexing gain. However, if the user decides to reduce the multiplexing gain to a smaller value $0<j<i$, then the probability of the outage depends on the outage of the $j$ many channels corresponding to $j$ largest eigenvalues, instead of $i$ many of them. The channel quantizer is fixed and transmitter and receiver do not change the channel quantizer and power allocation. 
Thus, the effect of quantized power control $G(m, n, i, L)$ remains unchanged, even when the system operates at a lower multiplexing gain.

To elaborate the above discussion, Figure 4 depicts the diversity-multiplexing curve given in the Theorem 6 for a system with 1 bit of feedback, $m=3$, and $n=4$. Also for the sake of comparison the diversity-multiplexing curve of a system without feedback, as given in [8], is plotted. There are three sets of curves in Figure 4. The solid curve, dashed curves with circle markings, and dashed with triangle. The dashed curve with triangle markings at the bottom is the diversity-multiplexing curve of a system without feedback. The solid envelope is the maximum diversity-multiplexing, which is the max operation in (33). The discontinuities at the integer multiplexing gains are due to the switching the channel quantizer to a smaller eigenvalue due to the operation at a smaller multiplexing gain for a fixed choice of channel quantizer. The set of dashed lines with circle markings can be divided into three curves. Since $m=3$, there are three eigenvalues that can be used to design the channel quantizer. The curve with highest slope that crosses the multiplexing axis at $r=1$ corresponds to the quantization of $\lambda_{3}$, which is the largest eigenvalue. The dotted curve with smaller slope that crosses the multiplexing axis at $r=2$ and has a discontinuity at $r=1$ corresponds to the quantization of $\lambda_{2}$, the second largest eigenvalue. When a multiplexing gain larger than 1 is desired, the outage is determined by the realization of $\lambda_{2}$, whereas for multiplexing gains smaller than one it suffices to receive data reliably on the channel corresponding to $\lambda_{3}$. Therefore, the outage behavior is determined by the realization of $\lambda_{3}$. An analytical description of the above is given in (46). Finally the dashed and dotted curve with the smallest slope (still with circle marking) that crosses the multiplexing axis at $r=3$ corresponds to the quantization of $\lambda_{1}$, which is the smallest eigenvalue. Similarly there are discontinuities at intermediate integer multiplexing gains of $r=1,2$.

Note that for any choice of eigenvalue $\lambda_{i}$ as channel parameter, for the multiplexing gain $r=i$, the diversity order is zero. This is the side effect of our view to the channel and achievable rate. When $i^{t h}$ largest eigenvalue is chosen to be the channel parameter, the channel is viewed as virtually $i$ parallel channel. To achieve a multiplexing gain of $r=i$ is the same as achieving a multiplexing gain of 1 from each of the virtual channels. Achieving a multiplexing gain of 1 on a channel with degrees of freedom of 1 is the same as achieving the ergodic capacity with finite block length, which is impossible. Therefore, at $r=i$ probability of error does not vanish with SNR, and diversity order does not exceed zero.

As the number of bits in feedback increases, the envelope curve stretches vertically (e.g. example 4 is an example of the effect of number of bits on the value of the diversity order). Hence, one can expect that as the number of feedback bits approaches infinity, i.e., perfect channel state information at the transmitter, the diversity gain approaches infinity for any arbitrary multiplexing gain, which complies with results in [11].

Figure 5 (a) shows the probability of outage of $2 \times 1$ system with $1, \log _{2}(3)$, and 2 bits of feedback $(L=2,3,4)$ with constant data rate of $2 \mathrm{bits} / \mathrm{s} / \mathrm{Hz}$. The solid curves are the outage found from numerical solution of (25). The dashed curves are the outage calculated based on the developed suboptimal power control scheme. Even though we showed the asymptotic optimality of our simplified scheme, Figure 5 also verifies our previous finding. Solid lines in Figure 5 (b) are diversity order for $L=3,4$ of the system with the same parameters as in (a). 


\subsection{Proof of Theorem 6}

We divide the proof of Theorem 6 into three steps. Two Lemmas, which are interesting results by themselves, provide the required tools for deriving the diversity order of quantized feedback. Body of the proof, which uses the results of the lemmas and takes care of the subtleties to prove the statement of the theorem.

As we explained in the beginning of Section 5, although conservative, in order to achieve multiplexing gain of $r=i$, we allocate power that guarantees a multiplexing gain of 1 on the channel corresponding to $\lambda_{i}$. Similarly, we define the outage, when the channel corresponding to the $\lambda_{i}$ is in outage, i.e., $\operatorname{Pr}\left\{\lambda_{i}<\gamma_{0}\right\}$. Therefore, in order to find the outage probability, distribution of $\lambda_{i}$ is needed. In Lemma 7 we find the distribution of $\lambda_{i}, 1 \leq i \leq m$.

The outage probability as given in (21) depends on $\gamma_{0}$, which is determined by the quantization and power allocation. Finding $\gamma_{0}$ requires solving the optimization problem (23). We find the quantizer and power allocation using the developed equi-power quantizer. The function $G(\cdot, \cdot, \cdot, \cdot)$ in the statement of the theorem is the effect of the quantized power control on the diversity order. In Lemma 8 we find the diversity order of quantized feedback when $\lambda_{i}$ is being fedback to the transmitter.

The rest of the proof deals with the technicality of finding the maximum diversity order for a given multiplexing gain, which explains having "max" in the statement of the Theorem 6 . The argument is based on the idea of having the larger eigenvalue as the channel parameter results in a smaller outage probability and hence larger diversity order.

The distribution of the ordered eigenvalues are unknown for the general case $\lambda_{i}$, and is only known for the smallest eigenvalue [20]. Since the diversity order is an asymptotic property, it suffices to know the asymptotic behavior of the distribution of $\lambda_{i}$, which is provided in the next lemma.

Lemma 7 (Asymptotic property of CDF of $\lambda_{i}$ ) Let $\lambda_{i}$ be the ith largest eigenvalue with probability distribution function $f_{\lambda_{i}}(x)$. Define $F_{\lambda_{i}}(t)$ by

$$
F_{\lambda_{i}}(t)=\int_{0}^{t} f_{\lambda_{i}}(x) d x
$$

then the Taylor expansion of $F_{\lambda_{i}}(t)$ around the origin is given by

$$
F_{\lambda_{i}}(t)=\beta_{i} t^{(n-i+1)(m-i+1)}+o\left(t^{l}\right),
$$

for some $l>(n-i+1)(m-i+1)$.

Proof: Consider the ordered statistics of the eigenvalues of the matrix $Z$. The distribution of the ordered eigenvalues of Wishart matrix, $\lambda_{m}>\lambda_{m-1}>\cdots>\lambda_{1}>0$, is given in [20]

$$
f\left(\lambda_{m}, \lambda_{m-1}, \ldots, \lambda_{1}\right)=e^{-\sum_{i=1}^{m} \lambda_{i}} \prod_{i=1}^{m} \lambda_{i}^{n-m} \prod_{i<j}\left(\lambda_{i}-\lambda_{j}\right)^{2},
$$

where $m=\min (M, N)$ and $n=\max (M, N)$.

While our analysis is based on the $i$ th largest eigenvalue, for simplicity of the proof we study the asymptotic properties of the $i$ th smallest eigenvalue. The conversion can be done by change of indexing, that is the $i$ th smallest eigenvalue is $m-i+1$ st largest. 
Let the joint distribution of the $\lambda_{i}$ 's, $1 \leq i \leq m$, be given by Equation (36). Then the marginal distribution of $\lambda_{i}$ 's, can be written as

$$
\begin{gathered}
f_{\lambda_{i}}(t)=\int_{\lambda_{m-1}}^{\infty} \int_{\lambda_{m-2}}^{\lambda_{m}} \cdots \int_{\lambda_{i}}^{\lambda_{i+2}} \int_{\lambda_{i-2}}^{t} \cdots \int_{\lambda_{1}}^{\lambda_{3}} \int_{0}^{\lambda_{2}} f\left(\lambda_{m}, \lambda_{m-1}, \ldots, \lambda_{1}\right) \\
d \lambda_{1} d \lambda_{2} \ldots d \lambda_{i-1} d \lambda_{i+1} \ldots d \lambda_{m-1} d \lambda_{m} .
\end{gathered}
$$

Note that the integration over $\lambda_{j}$ 's for $j>i$ results in a constant and is not a function of $\lambda_{i}$. On the other hand, evaluation of $f_{\lambda_{i}}(t)$ involves $(i-1)$ integrations over $\lambda_{j}$ 's, $1 \leq j<i$, each contributing a factor of $\lambda_{i}^{n-m+1}$ to the marginal distribution. ${ }^{5}$ Also for every $j$ and $k$ smaller than $i$, since $\lambda_{i}$ is assumed to be close to the origin, so are $\lambda_{j}$ and $\lambda_{k}\left(\lambda_{j, k}<\lambda_{i}\right)$. Hence, we can approximate $\left(\lambda_{k}-\lambda_{j}\right)^{2}$ for $j<k<i$ with $\lambda_{k}^{2}$, which results in a factor of $\lambda_{i}^{2(1+2+\cdots+(i-1))}$. Therefore, $f_{\lambda_{i}}(t)$ is of form

$$
f_{\lambda_{i}}(t)=t^{(n-m)+(i-1)(n-m+1)+2(1+2+\cdots+(i-1))} q(t) e^{-t}
$$

where $q(t)$ is a function of $t$ containing polynomials and exponential functions of $\lambda_{i}$ such that $q(0) \neq 0$. Let $k$ be the exponent of $x$ in Equation (37). Since $\frac{d F_{\lambda_{i}}(t)}{d t}=f_{\lambda_{i}}(t)$, the first $k$ derivatives of $\left.F_{\lambda_{i}}(t)\right)$ evaluated at 0 are equal to 0 . Thus, the Taylor expansion of $F_{\lambda_{i}}(t)$ around the origin can be written as

$$
F_{\lambda_{i}}(t)=\beta_{i} t^{k+1}+o\left(t^{l}\right)
$$

where $k+1=i(n-m+i)$ and some $l>i(n-m+i)$. Note that from (34) we have $F_{\lambda_{i}}(0)=0$. Therefore, there is no constant term in (38). By change of variable of $i$ to $m-i+1$ in Equation (38) we have the expression for the $i$ th largest eigenvalue

$$
F_{\lambda_{i}}(t)=\beta_{i} t^{(n-i+1)(m-i+1)}+o\left(t^{l}\right),
$$

for some $l>(n-i+1)(m-i+1)$.

As $P_{a v}$ increases, in order to meet the average power constraint (22), $P_{0}$ must increases, or equivalently $\gamma_{0}$ should decrease. Therefore, as $P_{a v}$ approaches infinity, $\gamma_{0}$ approaches zero. Using the Lemma 7, the outage expression (21) as $P_{a v} \rightarrow \infty$ can be reduced to

$$
\Pi\left(P_{a v}, R\left(P_{a v}\right)\right) \approx \begin{cases}\gamma_{0}^{(n-i+1)(m-i+1)} & \text { MIMO } \\ \gamma_{0}^{n} & \text { MISO } / \text { SIMO } \\ \gamma_{0} & \text { SISO. }\end{cases}
$$

To quantify the diversity order of power control with finite rate feedback, we need to formulate the probability of outage as a function of $P_{a v}$. A closer look at (39) one can notice that $\gamma_{0}$ is related to power allocated to the first quantization bin $P_{0}$, and $P_{0}$ in turn is related to the $P_{a v}$ through the average power constraint (22). Using the developed equi-power quantizer, we need to solve $L$ nonlinear transcendental equations of form (30) in order to find a closed form solution for $\gamma_{0}$. At large $P_{a v}$, a tight and accurate approximation to the equations (30) makes it possible to find $\gamma_{0}$ as a function of $P_{a v}$. The derivation is explained in next lemma.

Lemma 8 Consider a quantized power control with $L$ power levels which quantizes the ith largest eigenvalue of channel matrix. Define

$$
G(m, n, i, L) \triangleq \sum_{l=0}^{L-1}[(n-i+1)(m-i+1)]^{l} .
$$

\footnotetext{
${ }^{5} \int_{0}^{x} y^{\alpha} e^{-y} d y \approx y^{\alpha+1}$, for small $x$
} 
Then for large values of $P_{a v}$ the outage threshold $\gamma_{0}$ is given by

$$
\gamma_{0} \approx \frac{c_{i}}{P_{a v}^{\left(1-\frac{r}{i}\right) G(m, n, i, L)}}
$$

for multiplexing gain $r, 0 \leq r \leq i$, and some constant $c_{i}$.

Proof: Analyzing the suboptimum quantizer, with recursive equations explained in Section 4.3, with equal total power at each quantization bin, and beginning from the equation for the last quantization bin (30)we have

$$
\begin{aligned}
\frac{P_{a v}}{L} & =P_{L-1} \operatorname{Pr}\left\{\gamma_{L-1}<\lambda_{i}<\infty\right\} \\
& \stackrel{a}{=} \frac{P_{a v}^{r / i}}{\gamma_{L-1}}\left[1-F_{\lambda_{i}}\left(\gamma_{L-1}\right)\right]
\end{aligned}
$$

where (a) is obtained by replacing power allocated to the $L^{t h}$ bin, $P_{L-1}$, by its channel inversion equivalent. Solving (41) we have

$$
\gamma_{L-1} \approx \frac{c_{L-1}}{P_{a v}^{1-r / i}}
$$

Note that having $\gamma_{L-1}$ from (42), the expression of power allocated at the $L-1^{\text {st }}$ bin given by

$$
\begin{aligned}
\frac{P_{a v}}{L} & =P_{L-2} \operatorname{Pr}\left\{\gamma_{L-2}<\lambda_{i}<\gamma_{L-1}\right\} \\
& =\frac{P_{a v}^{r / i}}{\gamma_{L-2}}\left[F_{\lambda_{i}}\left(\gamma_{L-1}\right)-F_{\lambda_{i}}\left(\gamma_{L-2}\right)\right] \\
& \stackrel{a}{\approx} \frac{P_{a v}^{r / i}}{\gamma_{L-2}} F_{\lambda_{i}}\left(\gamma_{L-1}\right) \\
& \stackrel{b}{=} \frac{P_{a v}^{r / i}}{\gamma_{L-2}}\left(\frac{c_{L-1}}{P_{a v}^{1-r / i}}\right)^{(n-i+1)(m-i+1)}
\end{aligned}
$$

where (a) is obtained by neglecting $F_{\lambda_{i}}\left(\gamma_{L-2}\right)$ in comparison with $F_{\lambda_{i}}\left(\gamma_{L-1}\right)$ and (b) is obtained by combining (41) and (35). Note that (43) is only a function of $\gamma_{L-2}$, solving for $\gamma_{L-2}$ yeilds

$$
\gamma_{L-2}=\frac{c_{L-2}}{P_{a v}^{[1+(n-i+1)(m-i+1)]}}
$$

Repeating the procedure for all the bins sequentially toward the first bin yields the statement of the lemma.

So far the outage as a function of $\gamma_{0}$ and $\gamma_{0}$ as a function of $P_{a v}$ is derived. In order to complete the discussion it is needed to find the relation between $\lambda_{i}$ and the multiplexing gain. To do so, let revisit the expression for mutual information with quantized feedback (20)

$$
I(S ; Y \mid Q(H))=\sum_{k=1}^{m} \log \left(1+\frac{P\left(\lambda_{i}\right)}{M} \lambda_{k}\right) .
$$


Assume that a diversity order of $r$ is desired. If the $j^{\text {th }}$ term, $j=\lceil r\rceil$, in (44) is given enough power, then the achieved rate is larger than $j \log \left(1+P\left(\lambda_{j}\right) \lambda_{j} / M\right)$. Because, there are $j-1$ term preceding the $j^{\text {th }}$ term all of which have larger channel coefficient $\lambda_{k}, k<j$. That is equivalent of having a multiplexing gain of $j \geq r$. If we assume that the $j^{\text {th }}$ term is of form $(r / j) \log \left(1+P\left(\lambda_{i}\right) \lambda_{i} / M\right)$ and allocate power based on this rate, then the multiplexing gain of $r$ is guaranteed.

Similarly if a multiplexing gain of $r$ is desired, and $\left.\log \left(1+P\left(\lambda_{j}\right)\right) \lambda_{j}\right)<(r / j) \log \left(1+P_{a v}\right)$, then with high probability the transmission is in outage. Although we showed that outage only depends on the distribution of the $\lambda_{j}$ but $\lambda_{j}$ need not to be the channel parameter at the receiver that being quantized and fedback. For example, channel parameter could be $\lambda_{i}$, which determines the feedback and power levels at the transmitter, but a multiplexing gain $j<i$ is desirable. So the outage is determined by the distribution of $\lambda_{j}$, while $\gamma_{0}$ (the cutoff threshold) is determined by the average power and the distribution of $\lambda_{i}$.

The probability of outage can be obtained by replacing (40) into (39) and the diversity order of a MIMO system with $L$ level power control can be derived

$$
d=\left(1-\frac{r}{i}\right)(n-i+1)(m-i+1) G(m, n, i, L) .
$$

Note that the channel parameter $\lambda_{i}$ is being fedback, and the outage in (21) depends on the distribution of $\lambda_{i}$. Consider that a multiplexing gain of $j-1 \leq \eta<j$, with $j \leq i-1$ is desired. Then only the first $j$ terms (instead of $i$ terms) in (44) is needed to achieve the rate. Hence, the outage is determined by the distribution of $\lambda_{j}$, i.e.,

$$
\begin{aligned}
\Pi\left(R\left(P_{a v}\right)\right) & =\operatorname{Pr}\left\{\lambda_{j}<\gamma_{0 i}\right\} \\
& =\gamma_{0 i}^{(n-j+1)(m-j+1)} \\
& =\left(\frac{c}{P_{a v}^{(1-\alpha) G(m, n, i, L)}}\right)^{(n-j+1)(m-j+1)}
\end{aligned}
$$

for some constant $c$. Note that outage threshold is denoted as $\gamma_{0 i}$ to remind that it depends on the quantization parameter $\lambda_{i}$ and prevent confusion with $\lambda_{j}$.

\section{Extensions and Discussion}

\subsection{Joint Power and Rate Control}

In addition to power control, the finite feedback can be used for rate control or a combination of rate and power control. In this section we investigate the effect of power and rate control by constructing an example and analyzing its diversity and multiplexing tradeoff curve.

Let $\gamma_{t h}$ be a threshold on the channel state parameter, $\lambda_{i}$, such that a) $\gamma_{t h}$ vanishes as $P_{a v}$ increases, and b) for channel states $\lambda_{i}>\gamma_{t h}$ we use a codebook, $\mathcal{C}_{1}$, with rate $R_{1}\left(P_{a v}\right)=r_{1} \log \left(\alpha P_{a v}\right)$ and power $\alpha P_{a v}$, where $\alpha$ is a fixed constant (for all $P_{a v}$ 's) between zero and one, and c) for channel states $\lambda_{i}<\gamma_{t h}$ we use a codebook, $\mathcal{C}_{2}$, with rate $R_{2}\left(P_{a v}\right)=r_{2} \log \left((1-\alpha) P_{a v}\right)$.

Since $\gamma_{t h}$ decreases as $P_{a v}$ increase, asymptotically only codebook $\mathcal{C}_{1}$ is being used, i.e, the multiplexing gain is provided by the $\mathcal{C}_{1}$. A power allocation that guarantees outage free transmission of $\mathcal{C}_{1}$ fundamentally separates the outage event from the multiplexing achieving code. Using the rest of the quantization bins on quantized power control for $\mathcal{C}_{2}$ minimize the outage. However, as it is 
discussed in Section 4 outage is inevitable due to singularity of channel inversion at $\lambda_{i}=0$. Having $r_{1}<m$ guarantees an outage probability that decays with SNR with a slope greater than zero. The above intuition is summarized in the next theorem.

Theorem 9 (Diversity and multiplexing with power and rate control) A multiple antenna system with power and rate control described above has diversity order and multiplexing gain given by

$$
\begin{gathered}
d=\left(1-\frac{r_{1}}{i}\right)(n-i+1)(m-i+1) G(m, n, i, L-1) \\
0 \leq r \leq i .
\end{gathered}
$$

Proof: As $P_{a v}$ increases, the $\gamma_{t h}$ decreases. Therefore, the asymptotic rate is

$$
\begin{aligned}
R\left(P_{a v}\right) & =r_{1} \log \left(\alpha P_{a v}\right) \operatorname{Pr}\left\{\lambda_{i}>\gamma_{t h}\right\}+r_{1} \log \left((1-\alpha) P_{a v}\right) \operatorname{Pr}\left\{\lambda_{i}<\gamma_{t h}\right\} \\
& =r_{1} \log \left(\alpha P_{a v}\right) .
\end{aligned}
$$

Thus, the multiplexing gain of such a system depends only on the rate of $\mathcal{C}_{1}$. If we choose $\gamma_{t h}$ such that the power allocated to the $\mathcal{C}_{1}, \alpha P_{a v}$, guarantees a zero outage transmission, then the outage only happens in the transmission of codeword of $\mathcal{C}_{2}$. Therefore, the diversity order is the same as developed in the previous section, with the difference that the multiplexing gain is decoupled from the diversity order. Hence, $\mathcal{C}_{2}$ can have a fixed rate, zero multiplexing. Therefore, for $r=i, \mathcal{C}_{1}$ achieves the highest multiplexing gain, while $\mathcal{C}_{2}$ provides a non-zero diversity order. Note that in order to guarantee zero outage during the transmission of $\mathcal{C}_{2}$, the rate of the code can not exceed

$$
R\left(P_{a v}\right)=r \log \left(1+\alpha P_{a v} \gamma_{t h}\right) .
$$

and throughput is given by

$$
T\left(P_{a v}\right)=r \log \left(1+\alpha P_{a v} \gamma_{t h}\right) \operatorname{Pr}\left\{\lambda_{i}>\gamma_{t h}\right\} .
$$

Although we do not aim to choose a threshold $\gamma_{t h}$ that maximizes the throughput and we only care about the multiplexing gain, an approximation to the threshold that maximizes the total throughput given in (48) could be a good candidate for $\gamma_{t h}$. Since $\operatorname{Pr}\left(\gamma>\gamma_{t h}\right)$ is monotonically decreasing with $\gamma_{t h}$ and $\log \left(1+\alpha P_{a v} \gamma_{t h}\right)$ is monotonically increasing with $\gamma_{t h}$, there is a $\gamma_{t h} \in \mathcal{R}^{+}$that maximizes (48). Consider that $\gamma$ has the distribution of $\lambda_{i}$, for some $i, 1 \leq i \leq m$, and asymptotic approximation given in Lemma 7 , then by setting the first derivative of (48) with respect to $\gamma_{t h}$ to zero we have

$$
\frac{\alpha P_{a v}}{1+\alpha P_{a v} \gamma_{t h}}-\log \left(1+\alpha P_{a v} \gamma_{t h}\right) f\left(\gamma_{t h}\right)=0,
$$

where $f(\cdot)$ is probability density function of $\lambda_{i}$. Replacing $f\left(\gamma_{t h}\right)$ by approximation of (37) around the origin we get

$$
\gamma_{t h} \approx \frac{1}{\left(\log \left(\alpha P_{a v}\right)\right)^{1 / k}}
$$


where $k=(n-i+1)(m-i+1)$. Note that

$$
r_{i}=\lim _{\alpha P_{a v} \rightarrow \infty} \frac{\log \left(1+\frac{\alpha P_{a v}}{\left(\log \left(\alpha P_{a v}\right)\right)^{1 / k}}\right)}{\log \left(\alpha P_{a v}\right)}=r,
$$

as expected. Since for channel states greater than $\gamma_{t h}$ the channel can support higher rates, it is guaranteed that for this choice of threshold and power, the transmission of codewords from variable rate code is outage free. The diversity order however, is the same as in (32) with consideration that one of the quantization thresholds is used for rate control.

Figure 6 shows multiplexing-diversity curve derived from the results of Theorem 9 along with multiplexing-diversity curve for a system with no CSI at the transmitter. It is assumed that $m=$ $\min (M, N)=2, n=\max (M, N)=3$, and there are $B=1$ bits of feedback (which corresponds to a quantizer with 2 bins). The three points on the (multiplexing, diversity) curve for the system with one bit of feedback are $(0,42),(1,6)$ and $(2,2)$. Note that for a system with full multiplexing with finite rate feedback a nonzero diversity order is achievable.

\subsection{Location-dependent Power Control}

Temporal power control is employed in many systems such that the power control commands, which direct the transmitter to use a certain power level, are independent of average SNR of the channel. Average SNR is related to spatial location of the receiver in relation to the transmitter and depend on the mobility of the mobile and/or the environment. This implies that the power control thresholds are independent of receiver location, which is a judicious design for mobile systems.

However, in newer applications which are using MIMO links for point-to-point stationary links [23], the average conditions of the links do not vary that rapidly. Thus, the two nodes in the system could adapt their power control commands based on their relative location or equivalently average channel conditions. This leads to a system where the feedback link depends on the forward link average conditions, leading to much lower outage probabilities compared to a system which always uses the same power control commands for all forward link conditions. We believe that control or side channel adaptation is fundamentally required to improve on current systems which either have no side information or have a non-adaptive side information.

\section{Conclusions}

In this paper we studied the effect of channel side information at the transmitter. There are four major messages of this paper. First outcome indicates that power control (allocation of power in time) is much more effective then beamforming (spatial power allocation). While the beamforming schemes has the same diversity order as systems without CSIT, even few bits of information at the transmitter have a significant increase on diversity order and reliability. Second implicit result of the paper is that with perfect channel state information with long term power control there is no tradeoff between multiplexing gain and diversity order. Except for special cases, for almost all configurations of antennas, either probability of outage has an exponential decay with SNR, or it is zero for finite SNR. Both cases have infinite diversity order. However, our third result of the paper, shows that the tradeoff does exist when quantized channel state information is available at the transmitter. That is, the diversity order with limited CSIT is finite, is a function of number of bits in feedback, and 
is much larger than diversity order of system with CSIR only. However, the diversity order of a quantized power control system is zero at the maximum multiplexing gain, and is independent of the

feedback rate. Our fourth result implies that by applying a rate control mechanism it is possible to achieve a non-zero diversity order at highest multiplexing gain.

\section{A Proof of Theorem 3}

Since the transmitter has no knowledge about the channel, it is inevitable to allocate same power $P=P_{a v} / M$ on all the transmit antennas. Therefore, the expression for mutual information can be written as

$$
\begin{aligned}
I(X ; Y) & =\log \operatorname{det}\left(I_{N}+\frac{P_{a v}}{M} H H^{\dagger}\right) \\
& \stackrel{S}{\underline{V} D} \log \operatorname{det}\left(I_{N}+\frac{P_{a v}}{M}\left(U \Lambda^{1 / 2} V^{\dagger}\right)\left(U \Lambda^{1 / 2} V^{\dagger}\right)^{\dagger}\right), \\
& =\log \operatorname{det}\left(I_{m}+\frac{P_{a v}}{M} \Lambda\right), \\
& =\log \left(\prod_{i=1}^{m}\left(1+\frac{P_{a v}}{M} \lambda_{i}\right)\right), \\
& =\log \left[\left(\frac{P_{a v}}{M}\right)^{m}\left(\prod_{i=1}^{m} \lambda_{i}+\frac{M}{P_{a v}} \sum_{i=1}^{m} \prod_{j \neq i} \lambda_{i}+\cdots+\left(\frac{M}{P_{a v}}\right)^{m}\right)\right]
\end{aligned}
$$

In the limit as $P_{a v} \rightarrow \infty$, the expression for mutual information in (52) is reduced to

$$
I(X ; Y)=\log \left[\left(\frac{P_{a v}}{M}\right)^{m} \prod_{i=1}^{m} \lambda_{i}\right]
$$

The probability of outage is

$$
\begin{aligned}
\Pi_{C S I R} & =\operatorname{Pr}\{I(X ; Y)<R\} \\
& =\operatorname{Pr}\left\{\log \left[\left(\frac{P_{a v}}{M}\right)^{m} \prod_{i=1}^{m} \lambda_{i}\right] \leq m \log P_{a v}\right\} \\
& =\operatorname{Pr}\left\{\prod_{i=1}^{m} \lambda_{i} \leq \frac{1}{M^{m}}\right\} .
\end{aligned}
$$

The outage probability is independent of $P_{a v}$ and is non-zero. Therefore, the total achievable rate can not exceed $\left(1-\Pi_{C S I R}\right) R=m\left(1-\Pi_{C S I R}\right) \log P_{a v}$. Thus the multiplexing gain is at most $m\left(1-\Pi_{C S I R}\right)$. 


\section{B Proof of Theorem 2}

The optimum power control for a MIMO system with long term power control is found in [19] and given by

$$
P_{i}=\left[\left(\frac{2^{R / m}}{\left(\Pi_{j=1}^{m} \lambda_{j}\right)^{1 / m}}\right)-\frac{1}{\lambda_{i}}\right]^{+} .
$$

There are some conditions regarding positiveness of $P_{i}$ which are not important in an asymptotic regime when $R$ can be assume large enough that $P_{i}$ 's are all positive for all $i \in\{1,2, \ldots, m\}$. Having $R=r \log \left(P_{a v}\right)$, the outage region of the optimum power control is defined as the channel realizations for which power is not allocated, i.e.

$$
\Omega_{o f f}=\left\{\Lambda: \mathbb{E}\left[\sum_{i=1}^{m} P_{i}\right]>P_{a v}\right\} .
$$

From (53) we have

$$
\begin{aligned}
P_{1} & =\left(\frac{P_{a v}^{r / m}}{\left(\Pi_{j=1}^{m} \lambda_{j}\right)^{1 / m}}\right)-\frac{1}{\lambda_{1}} \\
& <\frac{P_{a v}^{r / m}}{\lambda_{1}}-\frac{1}{\lambda_{1}} \\
& \approx \frac{P_{a v}^{r / m}}{\lambda_{1}} \quad \text { for } P_{a v} \text { sufficiently large }
\end{aligned}
$$

which is an approximation to the power corresponding to the largest eigenvalue (assuming ordered eigenvalues with $\lambda_{1}$ the largest). We can obtain a similar approximation for $P_{m}$, the power corresponding to the smallest eigenvalue, i.e.,

$$
P_{m} \approx \frac{P_{a v}^{r / m}}{\lambda_{m}}
$$

Using $P_{1}$ and $P_{m}$ define

$$
\underline{B}=\left\{\Lambda: \mathbb{E}\left\{m P_{1}\right\}>P_{a v}\right\} \text { and } \bar{B}=\left\{\Lambda: \mathbb{E}\left\{m P_{m}\right\}>P_{a v}\right\} .
$$

Then it is clear that

$$
\underline{B} \subseteq \Omega_{o f f} \subseteq \bar{B} .
$$

Therefore, probability of outage can be bounded by

$$
\operatorname{Pr}\{\underline{B}\} \leq \Pi\left(P_{a v}, R\left(P_{a v}\right)\right) \leq \operatorname{Pr}\{\bar{B}\} .
$$

Let $\gamma_{0}^{1}$ be the solution to

$$
\int_{\gamma_{0}^{1}}^{\infty} m P_{1} f_{\gamma_{1}}(x) d x=P_{a v}
$$


and $\gamma_{0}^{m}$ be the solution to

$$
\int_{\gamma_{0}^{m}}^{\infty} m P_{m} f_{\gamma_{m}}(x) d x=P_{a v}
$$

then inequalities in (57) can be expanded as

$$
\operatorname{Pr}\left\{\lambda_{1}<\gamma_{0}^{1}\right\} \leq \Pi\left(P_{a v}, R\left(P_{a v}\right)\right) \leq \operatorname{Pr}\left\{\lambda_{m}<\gamma_{0}^{m}\right\} .
$$

- Case 1: $(r<m)$

Replace the distribution of the smallest eigenvalue [20] and (54) in (59) and solving for $\gamma_{0}^{m}$ we have

$$
\begin{aligned}
P_{a v} & =\int_{\gamma_{0}^{m}}^{\infty} m \frac{P_{a v}^{r / m}}{x} \frac{x^{n-m} e^{-x}}{\Gamma(n-m+1)} d x \\
P_{a v}^{1-r / m} & =\frac{m}{\Gamma(n-m+1)} \int_{\gamma_{0}^{m}}^{\infty} x^{n-m-1} e^{-x} d x \\
& <\frac{m}{\Gamma(n-m+1)} \int_{0}^{\infty} x^{n-m-1} e^{-x} d x \\
& =\frac{m \Gamma(n-m)}{\Gamma(n-m+1)} \\
& =\frac{m}{n-m} .
\end{aligned}
$$

Therefore, for sufficiently large $P_{a v}$, to be specific for $P_{a v}>(m /(n-m))^{m /(r-m)}, \gamma_{0}^{m}$ is zero, which is equivalent of saying $\bar{B}=\{\}$, which in turn implies that $\operatorname{Pr}\{\bar{B}\}=0$. From (57) and asymptotic emptiness of $\bar{B}$ we conclude

$$
\begin{aligned}
d & >\lim _{P_{a v} \rightarrow \infty}-\frac{\log (\operatorname{Pr}\{\bar{B}\})}{\log \left(P_{a v}\right)} \\
& =\infty
\end{aligned}
$$

The argument is not yet complete and requires more careful consideration for $n=m$. For the case of $n=m$ look at the single antenna setting below.

- Case 2: $(r<m$ and $n=m)$

For a single antenna system or when number of transmit and receive antennas are equal, smallest (or the only) eigenvalue has the exponential distribution, i.e., $f_{\lambda_{m}}(x)=e^{-x}$. Solving (59) for $\gamma_{0}^{m}$ ) we have

$$
\begin{aligned}
P_{a v}^{1-r / m} & =\int_{\gamma_{0}^{m}}^{\infty} m \frac{e^{-x}}{x} d x \\
& \approx m \log (x) \quad \text { for small } \gamma_{0}^{m} .
\end{aligned}
$$


Therefore, $\gamma_{0}^{m} \approx e^{-P_{a v}^{1-r / m} / m}$. Now we can find $\operatorname{Pr}\{\bar{B}\}$ by

$$
\begin{aligned}
\operatorname{Pr}\{\bar{B}\} & =\operatorname{Pr}\left\{\lambda_{m}<\gamma_{0}^{m}\right\} \\
& =\int_{0}^{\gamma_{0}^{m}} e^{-x} d x \\
& =e^{-\gamma_{0}^{m}} \\
& =1-e^{-e^{-\frac{P_{a v}^{1-r / m}}{m}}} \\
& \approx e^{-\frac{P_{a v}^{1-r / m}}{m}}
\end{aligned}
$$

Which yields same diversity order as in (62)

- Case 3: $(r=m)$

From (63) and (61) it is immediate that $\gamma_{0}^{m}=c_{m}$, where $c_{m}$ is a constant. Thus, $\operatorname{Pr}\{\bar{B}\}$ is constant and independent of $P_{a v}$. If $\operatorname{Pr}\{\bar{B}\}$ is zero, then there is no outage, and a diversity order of infinity is achieved, but if $\operatorname{Pr}\{\bar{B}\}$ is not zero, then we need to find the $\operatorname{Pr}\{\underline{B}\}$.

Case of $n=m=1$ which is the single antenna case is treated in the previous case and it is shown that the probability of outage is constant, and hence the upper bound for the diversity order for a single antenna system is also zero. Thus the maximum diversity order for a single antenna system at maximum multiplexing gain $r=m$ is zero.

- Case 4:(r=m,n>1,m=1) This case includes MISO and SIMO systems. The channel is defined by a single scalar parameter which is the magnitude of the channel vector with gamma distribution. The boundary of the outage region can be found from the average power constraint, i.e.,

$$
\begin{aligned}
P_{a v} & =\int_{\gamma_{0}}^{\infty} \frac{P_{a v}}{x} \frac{x^{n-1} e^{-x}}{\gamma(n)} d x \\
1 & =\int_{\gamma_{0}}^{\infty} \frac{x^{n-2} e^{-x}}{\gamma(n)} d x .
\end{aligned}
$$

For $\gamma_{0}=0$ Equation (64) is reduced to $1=1 /(n-1)$, and the outage region is defined by all channel states $\lambda$ for which $1 /(n-1)>1$, which is an empty set. Therefore, the outage is zero and diversity order of infinity can be achieved at the maximum multiplexing gain of 1 .

- Case $5:(r=m, n, m>1)$

By having $r=m$ in (61) we get that for $n>2 m, \bar{B}$ is empty, and so is probability of outage, and the diversity order is infinite. However, for $m \leq n \leq 2 m$, on one hand $\bar{B}$ is not empty, and on the other hand the distribution of $\lambda_{1}$ is not known. So it is not clear that whether the probability of outage is zero or non-zero. We need to see if $\Omega_{o f f}$ is empty, and if it depends on $P_{a v}$.

For large values of $P_{a v}$, we can write the power corresponding to each eigenvalue in (53) as

$$
P_{i}=\frac{P_{a v}}{\left(\Pi_{j=1}^{m} \lambda_{j}\right)^{1 / m}}
$$


For large $P_{a v}$ all powers all almost equal and the term $1 / \lambda_{i}$ is negligible. The outage region, $\Omega_{o f f}$ is determined by the solution of $\mathbb{E}\left[m P_{i}\right]=P_{a v}$, for some $i$, i.e.,

$$
\mathbb{E}\left[\frac{1}{\left(\Pi_{j=1}^{m} \lambda_{j}\right)^{1 / m}}\right]=\frac{1}{m},
$$

which shows that the outage region does not depend on $P_{a v}$. Therefore, probability of outage is constant. If the outage region is empty, then probability of outage is zero and diversity order is infinite, and if outage region is non-empty, then diversity order is zero. Using Jensen's inequality we can further simplify (66) to get

$$
\begin{aligned}
\mathbb{E}\left[\frac{1}{\left(\Pi_{j=1}^{m} \lambda_{j}\right)^{1 / m}}\right] & \geq \mathbb{E}\left[\left(\Pi_{j=1}^{m} \lambda_{j}\right)^{1 / m}\right] \\
& =\mathbb{E}\left[\lambda_{j}\right],
\end{aligned}
$$

for an arbitrary unordered eigenvalue $\lambda_{j}$.

\section{References}

[1] I. E. Telatar, "Capacity of multi-antenna gaussian channels," AT\&T Bell Laboratories, Tech. Rep., June 1995.

[2] A. J. Goldsmith and P. P. Varaiya, "Capacity of fading channels with channel side information," IEEE Transactions on Information Theory, vol. 43, no. 6, pp. 1986-1992, November 1997.

[3] G. Caire, G. Taricco, and E. Biglieri, "Optimum power control over fading channels," IEEE Transactions on Information Theory, vol. 45, no. 5, pp. 1468-1489, July 1999.

[4] A.Narula, M. J. Lopez, M. D. Trott, and G. W. Wornell, "Efficient use of side information in multiple-antenna data transmission over fading channel," IEEE Jour. Selc. Area Comm., JSAC, vol. 16, no. 8, pp. 1423-1436, Oct. 1998.

[5] K. K. Mukkavilli, A. Sabharwal, E. Erkip, and B. Aazhang, "On beamforming with finite rate feedback in multiple antenna systems," IEEE Trans. Inform. Theory, vol. 49, no. 10, pp. 25622579, Oct. 2003.

[6] D. J. Love, R. W. H. Jr., and T. Strohmer, "Grassmannian beamforming for multiple-input multiple-output wireless systems," IEEE Trans. Infrom. Theory, vol. 49, no. 10, pp. 2735-2747, Oct. 2003.

[7] D. Rajan, "Power efficient transmission policies for multimedia traffic over wireless channels," Ph.D. dissertation, Rice University, Houston, TX, April 2002.

[8] L. Zheng and D. N. C. Tse, "Diversity and multiplexing: A fundamental tradeoff in multipleantenna channels," IEEE Trans. on Inform. Theory, vol. 49, no. 5, pp. 1073-1096, May 2003. 
[9] L. G-Ordóñez, A. P-Zamora, and J. Fonollosa, "Diversity and multiplexing tradeoff of multiple beamforming in MIMO channel," in IEEE Int. Symp. on Inform. Theory, ISIT'05, Adelaide, Australia, Sep. 4-9 2005.

[10] L. W. Hanlen and A. J. Grant, "Optimal transmit covariance for MIMO channels with statistical transmitter side informaiton," in IEEE Int. Symp. on Inform. Theory, ISIT'05, Adelaide, Australia, Sep. 4-9 2005.

[11] H. E. Gamal, G. Caire, and M. O. Damen, "The diversity-multiplexing-delay tradeoff in MIMO ARQ channels," in IEEE Int. Symp. on Inform. Theory, ISIT'05, Adelaide, Australia, Sep 4-9 2005.

[12] D. Rajan, A. Sabharwal, and B. Aazhang, "Outage behaviour with delay and CSIT," in IEEE Int. Conf. on Communications, ICC'04, June 20-24 2004, pp. 578-582.

[13] C. Steger, A. Khoshnevis, A. Sabharwal, and B. Aazhang, "The importnace of transmitter side information and the value of training," in Proceedings of $42^{\text {nd }}$ Allerton Conference on Communication, Control, and Computing, Monticello, IL, Sep. 28-30 2005.

[14] A. Khoshnevis and A. Sabharwal, "Performance of quantized power control in multiple antenna systems," in IEEE Int. Conf. on Communications, ICC'04, Paris, France, June 20-24 2004, pp. 803-807.

[15] — _ "Achievable diversity and multiplexing in multiple antenna systems with quantized power control," in IEEE International Conference on Communication, ICC'05, Seoul, S. Korea, May 16-20 2005 .

[16] L. Lin, R. D. Yates, and P. Spasojevic, "Adaptive transmission with discrete code rates and power levels," IEEE Trans. on Communications, vol. 49, no. 5, pp. 1073-1096, May 2003.

[17] A. Khoshnevis and A. Sabharwal, "On diversity and multiplexing gain of multiple antenna systems with transmitter channel information," in Proceedings of $42^{\text {nd }}$ Allerton Conference on Communication, Control, and Computing, Monticello, IL, Sep 29-Oct 12004.

[18] L. H. Ozarow, S. S. (Shitz), and A. D. Wyner, "Information theoretic considerations for cellular mobile radio," IEEE Trans. Veh. Technol., vol. 43, no. 2, pp. 359-378, May 1994.

[19] E. Biglieri, G. Caire, and G. Taricco, "Limiting performance of block-fading channels with multiple antennas," IEEE Trans. Inform. Theory, vol. 47, no. 4, pp. 1273-1289, May 2001.

[20] A. Edelman, "Eigenvalues and condition numbers of random matrices," Ph.D. dissertation, Massachusetts Institute of Technology, MIT, May 1989.

[21] K. K. Mukkavilli, T. Muharemovic, A. Sabharwal, and B. Aazhang, "On the outage probability of a class of signaling schemes for multiple antennas," in IEEE Int. Symp. on Inform. Theory, ISIT'02, Lausanne, Switzerland, June 30-July 5 2002, p. 477.

[22] D. Faires and B. Faires, Calculus of One Variable, 2nd ed. Random House, 1989.

[23] R. Karrer, A. Sabharwal, and E. Knightly, "Enabling large-scale wireless broadband: The case for TAPs," in Proceedings of HotNets 2003, Cambridge, MA, Nov. 2003. 


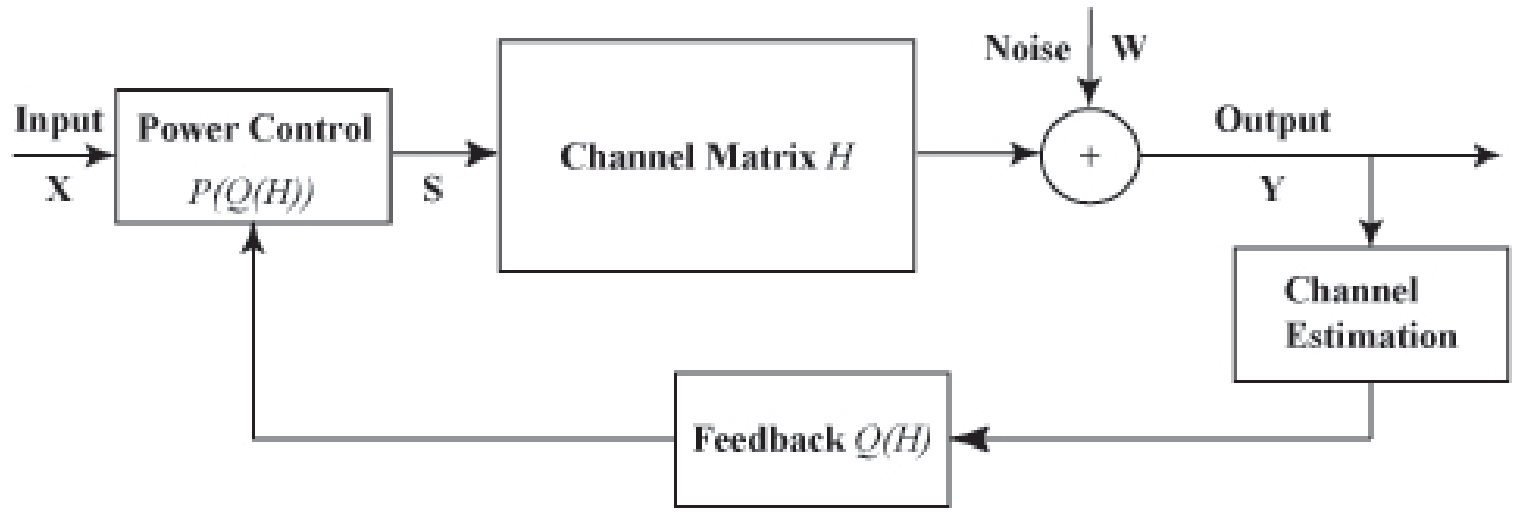

Figure 1: System with feedback. 


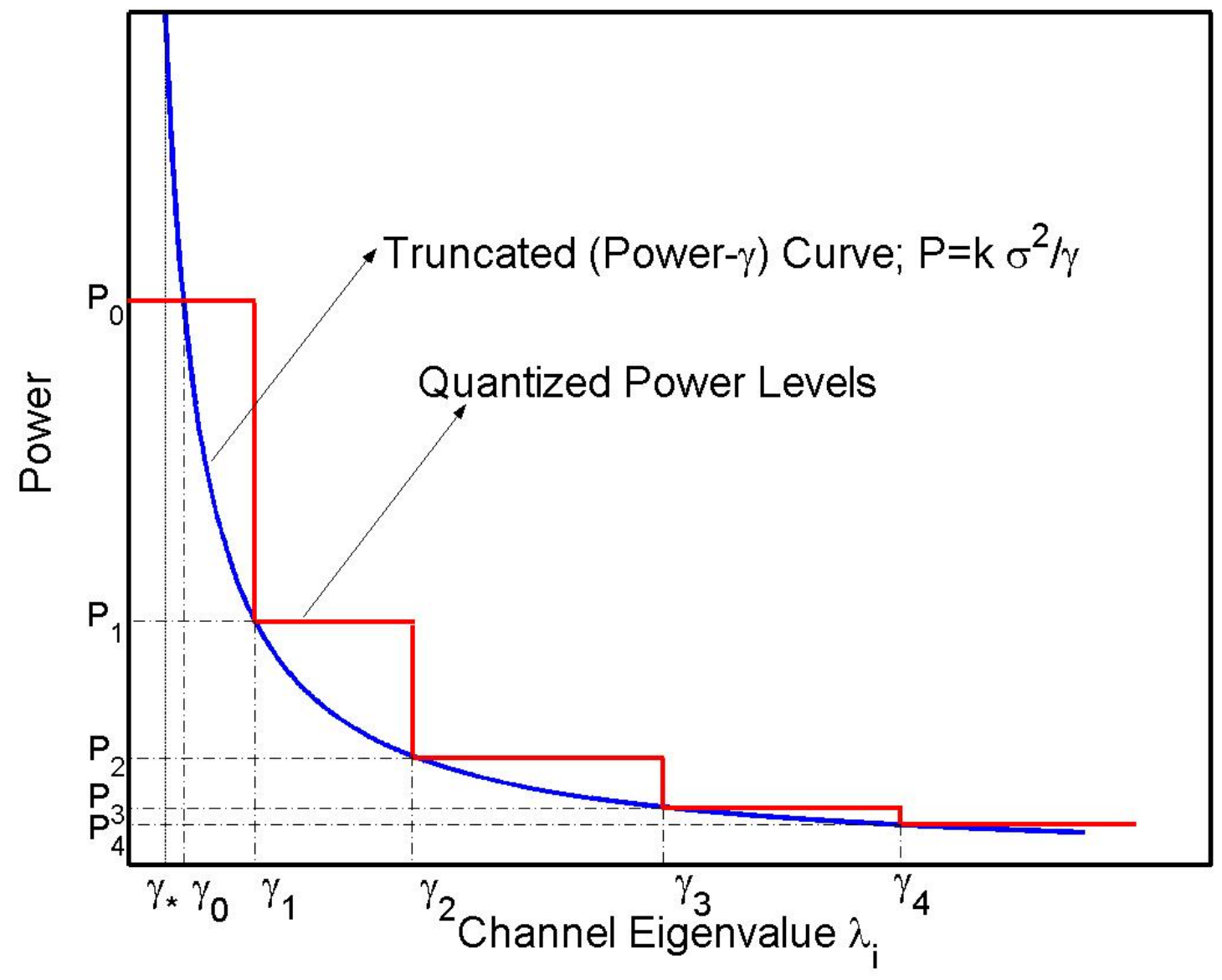

Figure 2: An example of a 5-bin channel quantizer with channel inversion power allocation. 


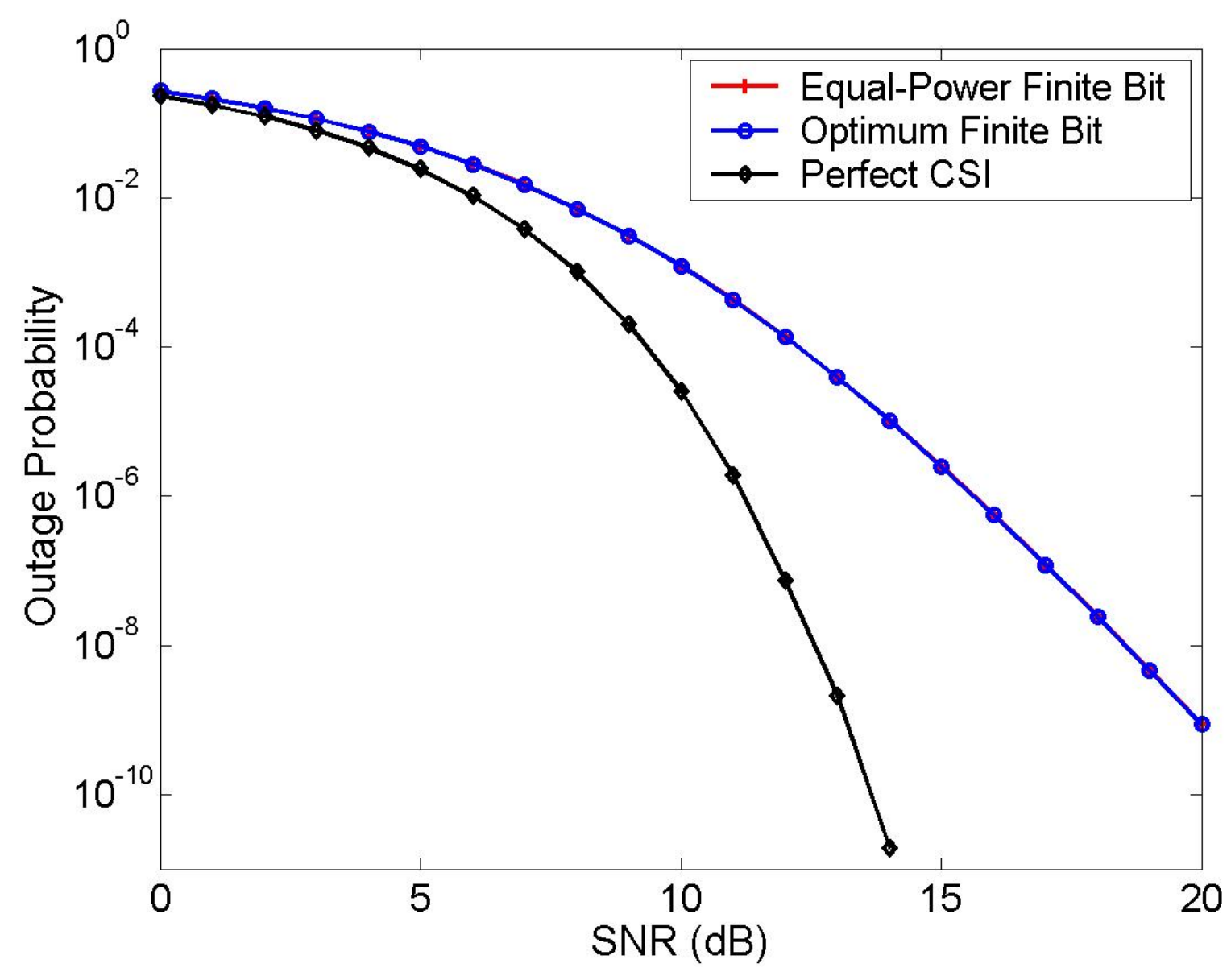

Figure 3: Outage for $\log _{2}(3)$ bits of feedback for optimal, equal allocation power control, and perfect CSI at Tx and Rx with respect to SNR for a system with single antenna at transmitter and receiver, and transmission rate of $\mathrm{R}=2 \mathrm{~b} / \mathrm{s} / \mathrm{Hz}$ 


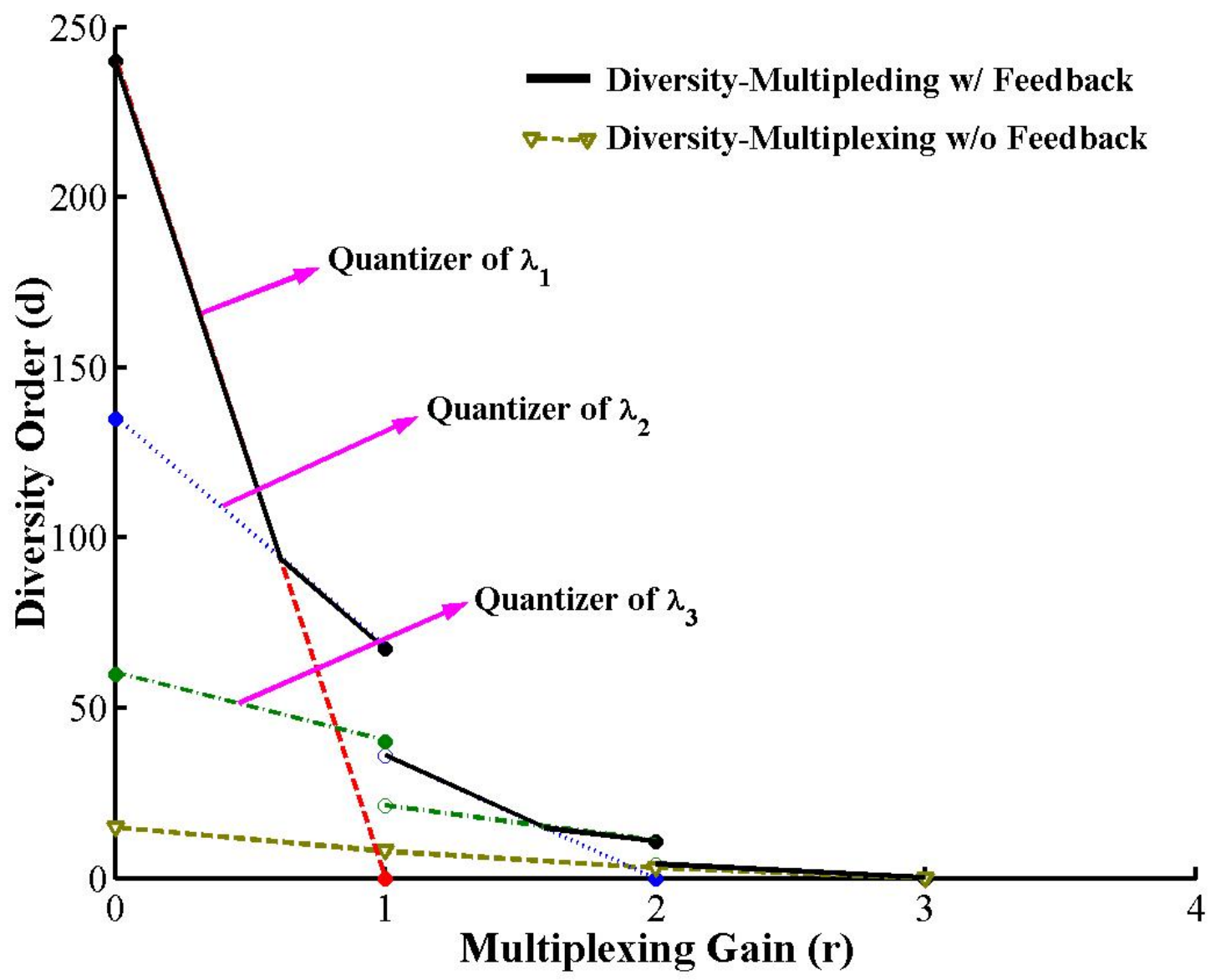

Figure 4: Diversity and Multiplexing curve with 1 bit of feedback and without feedback as in [8], for system with $m=3, n=5$. 


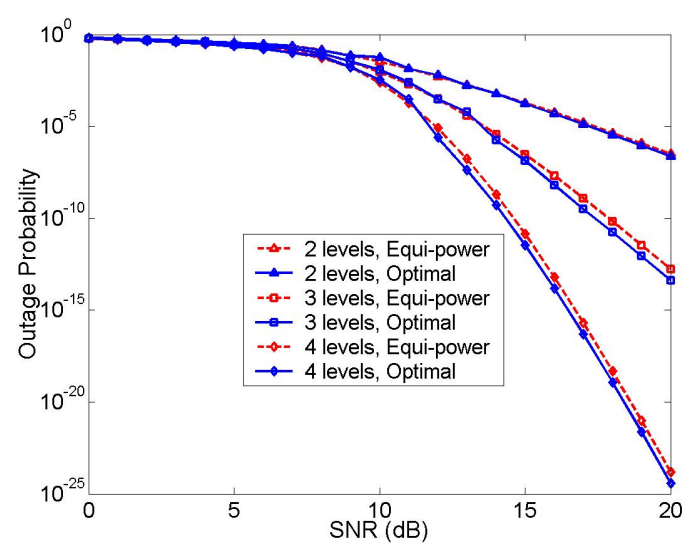

(a)

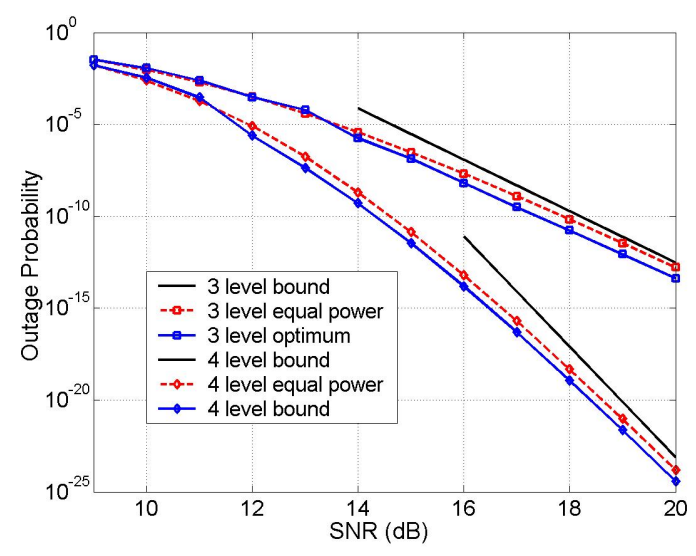

(b)

Figure 5: (a) Outage probability as a function of SNR for a system with $L=2,3,4$, optimum solution of (25) with developed suboptimal recursive solution, (b) bound on diversity order in corollary 6 for $\mathrm{L}=3$, and 4 with optimal and suboptimal power allocation schemes. Both graph are for a system with 2 transmit antennas, and rate $\mathrm{R}=2 \mathrm{bit} / \mathrm{s} / \mathrm{Hz}$. 


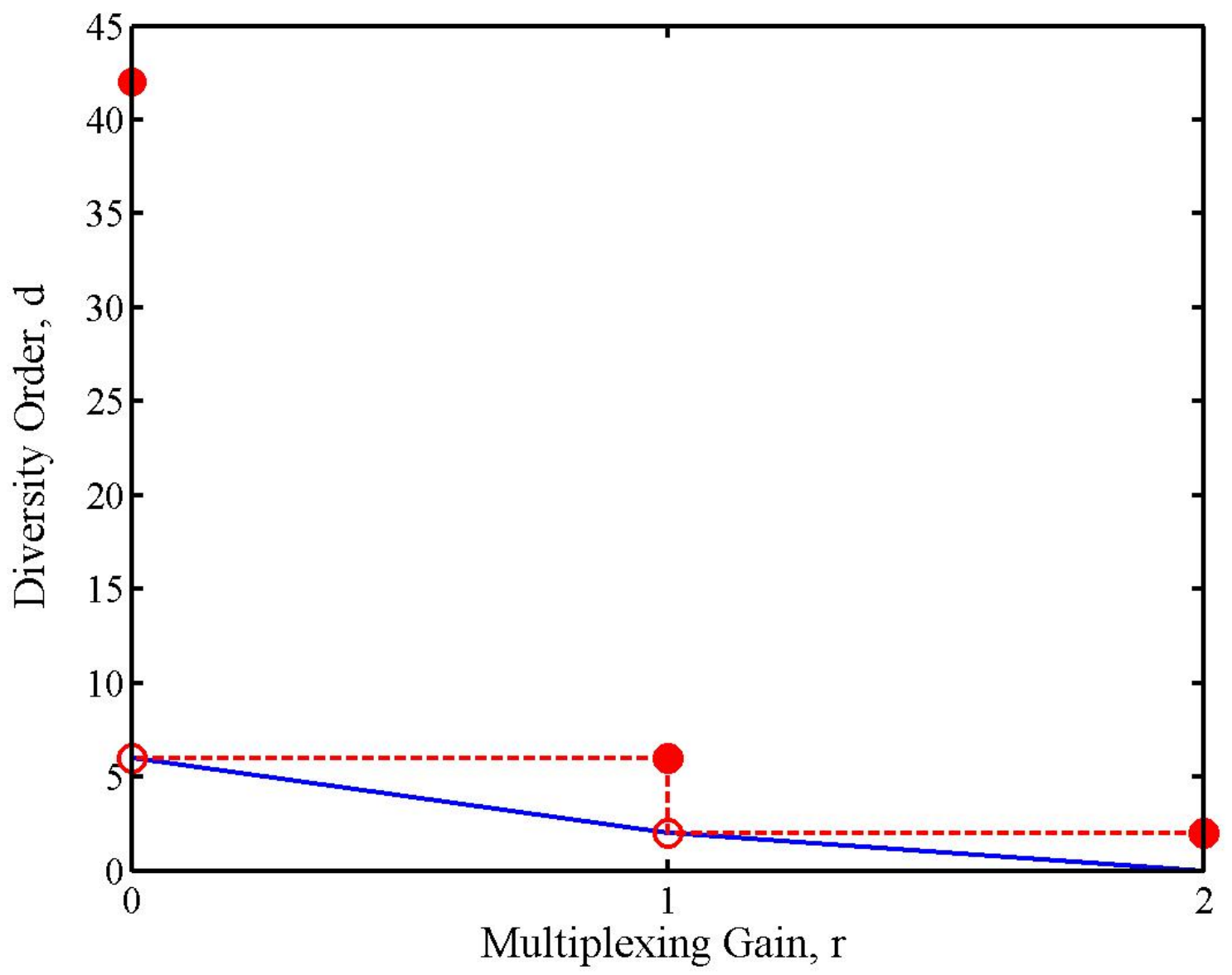

Figure 6: Diversity-Multiplexing curve for systems with no and partial CSI at the transmitter with $\mathrm{M}=2$ transmit and $\mathrm{N}=3$ receive antennas, and $\mathrm{B}=1$ bit of feedback. Solid curve is corresponding to the system with CSI only at the receiver. Dashed curve with circles corresponds to the system with perfect CSI at the receiver and partial knowledge at the transmitter. The filled circles and empty circles are corresponding to the closed and open end of the intervals respectively. 